\title{
Gear tooth failure detection by the resonance demodulation technique and the instantaneous power spectrum method - A comparative study
}

\author{
Ahmad Ghasemloonia ${ }^{\mathrm{a}, *}$ and Siamak Esmaeel Zadeh Khadem ${ }^{\mathrm{b}}$ \\ ${ }^{a}$ Faculty of Engineering, Memorial University, St. John's, NL, Canada \\ ${ }^{\mathrm{b}}$ Faculty of Engineering, Tarbiat Modares University, Tehran, Iran
}

Received 6 June 2007

Revised 6 November 2009

\begin{abstract}
The role of gears in industry for speed and torque variation purposes is obvious. The gearbox diagnostic methods have been improved quickly in recent years. In this paper, two of the newest methods, the resonance demodulation technique (R.D), and the instantaneous power spectrum technique (IPS) are applied to gearbox vibration signals and their capabilities in fault detection are compared. Yet, the important role of time averaging should not be dispensed with, as it is the primary step for both techniques. In the present study, the mathematical method of these techniques, according to the mathematical vibration model of gears, is introduced, these techniques are applied to the test rig data, and finally the results of both methods are compared. The results indicate that in each method, the location of fault can be estimated and it is located in the same angular position in both methods. The IPS method is applicable to severe faults, whereas the resonance demodulation technique is a simple tool to recognize the fault at each severity and at the early stages of fault generation.
\end{abstract}

Keywords: Gearbox, vibration analysis, resonance demodulation, instantaneous power spectrum, failure detection

\section{Introduction}

Vibration analysis has an old antecedent in monitoring and fault diagnosis of machinery. The gears and gearboxes are mostly used for special purposes in industry. Therefore, their fault diagnostics and monitoring techniques have been improved quickly. Various kinds of processing techniques can be grouped into five major categories: 1) raw signals, 2) time synchronous signal averaging, 3) residual signal, 4) difference signal and 5) band pass mesh signal. Localized gear defects have been extensively studied, since gear faults are mostly initiated by localized defects. Fatigue fracture and cracks are two samples of localized gear faults. In this paper, two methods of fault detection in gearboxes - the resonance demodulation technique and the instantaneous power spectrum- are studied and compared.

Mostly, defects alter the amplitude and phase of the gear vibration. Therefore, vibration monitoring for gearbox fault detection using different methods have been improved [12]. At the end of nineties, the time-frequency techniques were widely used for gears fault detection. Different kind of time-frequency distribution methods, such as wavelet transform, Wigner-Ville distribution, Pseudo Wigner-Ville distribution were applied by Forrester, McFadden, Wang,

*Corresponding author: Ahmad Ghasemloonia, Box \#60, Faculty of Engineering and Applied Science, Memorial University of Newfoundland, St. John's, NL, Canada A1B 3X5. Tel.: +1 709 7495027; E-mail: a.ghasemloonia@mun.ca. 
Baydar, Staszewski and Yesilyurt [6,14,15,26,1,24,27] respectively. In one of the recent works by Wang et al. [25], the sensitivity of the phase and amplitude modulation techniques and wavelet transform were studied. The results show that the beta Kurtosis factor is a reliable tool for gear diagnostic. Smoothed Pseudo-Wigner-Ville distribution of an acoustic signal was used as a tool for local fault detection in gearboxes by Baydar and Ball [2]. They suggested the acoustic signal as an effective tool for gearbox diagnostics in the early stages of fault generation. Mohanty and Kar [18] studied the motor current wave form as a tool in the fault detection of multistage gearbox. They used the decomposed frequency demodulated current of the induction motors, which drives the gearbox, to monitor different frequency levels in the gearbox.

A full review of vibration processing techniques for gear fault detection such as time- frequency and cyclostationarity analysis was performed by Dalpiaz et al. [5] and the results have been compared to cepstrum analysis and timesynchronous averaging analysis results for different depth of crack. Moreover, the effects of different positioning places of transducer on the gear box case were shown. In this paper, the residual signal and demodulation techniques were suggested as a well known tool for diagnosis depending on a proper filtering. Another complete review of diagnostic methods for helicopter transmission systems is presented by Samuel and Pines [21]. Their study covers a broad range of methods in Health and Usage Monitoring (HUM) systems like statistic criteria, time frequency distribution methods, Wavelet analysis as a joint time- frequency distribution, neural networks, and mathematical modeling of vibration data. They also included the performance assessment of presented diagnostic techniques and suggested more improvement in the field of waveform modeling and sensor development as well as signal processing methods.

Defects in the gear affect the instantaneous energy and frequency components in the modulation process. As the frequency increases, defects will appear in the frequency spectrum as sidebands, but not in lower frequencies [1]. In detecting the defects by vibration analysis, two important parameters are tooth meshing frequency (including its harmonics) and the sidebands. When a localized fault, such as a crack in a tooth mates with another tooth in a gearbox, it produces modulation effects and sidebands [16]. The interval of the sidebands and their amplitude mostly indicates a faulty condition. It is difficult to detect a localized fault in the spectral analysis method, due to difficulties in detecting corresponding fault sidebands in the presence of several gears in pair and other mechanical components, which also produce extra sidebands. Therefore, other vibration analysis methods are suggested for gear fault detection, such as time-synchronous averaging, time-frequency distribution, signal modeling techniques, cepstrum and statistical methods $[10,14]$.

In the time domain analysis, time synchronous averaging of the raw vibration signal removes periodic events related to the non-faulty gears and also reduces the noise effects. Therefore, processing techniques of the time averaging method, such as the extraction of the residual signal, amplitude and phase modulation of a tooth meshing harmonics, have been improved for early detection of gear damages. Consequently, because of the impacts produced by local faults in mating tooth, the vibration signal of the faulty gearbox is considered as a non-stationary signal [26]. Thus, the methods which are based on the analysis of stationary signals are not suitable for gear fault detections. On the other hand, the application of time-frequency distribution methods, such as wavelet transform, is useful in the time localizing of events and detecting cracks in a special gear. Halim et al. [8] combined two methods of time synchronous averaging and wavelet transformation, and presented a new method called time domain averaging across all scales. He verified that removing noise and periodic events from the characteristic vibration signal of a faulty gearbox is an effective step in fault detection, and this method facilitates this feature by capturing dynamic characteristics of one period of the vibration signal.

In the frequency spectrum of a non-faulty gearbox, low order modulation sidebands are appearing around low order mesh frequencies and its harmonics. Due to the impact feature of gear faults in a complete revolution in a faulty gear, higher order sidebands spread over a wide range of frequencies around high order mesh harmonics. For the purpose of fault detection, the signal should be averaged. This averaging reduces the noise effect and removes regular gear meshing harmonics. If the gear meshing harmonics are removed from the averaged signal, the result is the residual signal. The residual signal involves some information about faults [12]. Impact resonate the structure of the gear and this resonance plays an amplifying role for weak defects. Sidebands around low order mesh harmonics are generated due to signal leakage and geometrical errors of gear and should not be taken into account. Therefore, in a frequency spectrum of a faulty gear, sidebands around a high order mesh harmonic are of great concern. Using a "Chebyshev" type2 filter designed in "sptool" toolbox of Matlab ${ }^{\circledR}$, one may first implement band-pass filtering 
and then stop-band filtering, and then the residual signal is attained. This residual signal is then transformed into time-space and squared, where the Kurtosis factor of this squared signal is determined. Taking into account that the Kurtosis value higher than 4 indicates the faulty condition, the fault could be estimated [22]. A phase diagram indicates the angular position of a faulty tooth. Further in the paper, a mathematical model is simulated for gear vibration and using this model, the theoretical basis of the method is developed extensively.

Another method which is used in this study is a type of "Cohen-class" of "quadratic time-frequency distribution", which is called the instantaneous power spectrum (IPS) [4]. The application of this method is simpler than the RD technique, but, as will be seen, this method does not have the ability of fault detection in the early stages of fault generation. This method indicates to the presence of fault as a criterion of energy concentration. In fact, the faults are revealed by cumulating energy spots on a specific time and frequency on the IPS contour plots. This method was first applied by Page [19] and then was completed by Levin [11]; eventually it was extended by Hippenstiel and Olivera [9]. But, due to the ringing effect, prediction of fault for a noisy signal is difficult, in spite of reinforcement of the pertinent signal features. According to the above facts, and considering that in early stages of fault generation the energy level is not comparable to noise energy, the prediction of fault presence in this stage is not simple. To apply this method, similar to R.D. technique, the signal should be averaged and, then, the two autocorrelation function should be added to each other over the averaged cycle. After that, this sum should be normalized and weighted. For weighting functions, window functions such as Kaiser Window are used, knowing that the IPS is not sensitive to window parameters such as type and length. The endpoints of windows are not deleted in this technique [20]. The FFT of the weighted autocorrelation sum is known as the IPS. The position of the fault also is available in the IPS contour plots. The mathematical basis of this method is explained in the next section. The results of two methods should demonstrate the same position of the fault in the gearbox and the same fault severity percentage.

\section{The mathematical basis of the resonance demodulation technique}

It is essential to introduce the signal model of gear vibration. In the case of ideal gears mating with rigid teeth and exact involute profiles, gears transmit uniform angular motion. In the case of real gears, any deviation of above ideal state will lead to variation in angular displacement and velocity, i.e., the transmission error [20]. Vibration, which is generated in gear mating, is transmitted through gear shaft bearing. Consider a pair of perfect gear mating under a constant load and speed and their stiffness varies periodically with the number of teeth in contact and the position on tooth surface. Vibration of this system will be excited at tooth meshing frequency and its harmonics [22]. At a complete revolution, after being synchronously averaged, the vibration of this pair of gears is approximately estimated in terms of gear meshing frequency and its harmonics by the following equation [7]:

$$
x(t)=\sum_{k=0}^{K} X_{k} \cos \left(2 \pi k f_{m} t+\varphi_{k}\right)
$$

Where $\mathrm{X}_{k}$ represents the amplitude at the kth frequency, $\mathrm{f}_{m}$ is the mesh frequency, $\mathrm{k}(0,1,2 \ldots, \mathrm{K})$ is the mesh harmonics and $\varphi_{k}$ is the initial phase. This is the case when the tooth profile error is not taken into account. This error changes the amplitude and phase of the vibration. If these changes are described by the phase and amplitude modulating functions $\left\{\mathrm{a}_{k}(\mathrm{t})\right.$ and $\left.\mathrm{b}_{k}(\mathrm{t})\right\}$, the complete signal model per revolution of gear is described as follows $[16$, 13]:

$$
x(t)=\sum_{k=0}^{K}\left[1+a_{k}(t)\right] X_{k} \cos \left[2 \pi k f_{m} t+\varphi_{k}+b_{k}(t)\right]
$$

The measured vibration average is a superposition of modulated tooth meshing harmonics. If a local fault is present, then an impact function should be added to the above equation. This impact will generate a structural resonance in the gear and this resonance has an amplifying role on low impacts. This impact function is [26]:

$$
i(t)=e(t) \cos \left(2 \pi f_{r} t+\theta_{r}\right)
$$


Where $\mathrm{e}(\mathrm{t})$ is the modulating function of the resonant vibration, $\mathrm{f}_{r}$ is the carrier frequency and $\theta_{r}$ is the initial phase. Therefore, in the case of a faulty gear, the impact function should be added to the Eq. (2). Finally, the signal model of the faulty gear vibration is as follows:

$$
y(t)=\sum_{k=0}^{K}\left[1+\tilde{a}_{k}(t)\right] X_{k} \cos \left[2 \pi k f_{m} t+\varphi_{k}+\tilde{b}_{k}(t)\right]+e(t) \cos \left(2 \pi f_{r} t+\theta_{r}\right)
$$

The impact introduces additional amplitude and phase modulation to the normal gear vibration which in the superposition function $y(t)$ appears as $\tilde{a}_{k}(t)$ and $\tilde{b}_{k}(t)$, denoting modified amplitude and phase modulation functions.

Now, having the signal model of gear vibration, in order to use resonance demodulation technique, the residual signal must be computed. The spectrum of the unmodulated vibration consists of a series of tooth meshing harmonics only. The modulated amplitude and phase are surrounded by modulation sidebands. Additionally, meshing harmonics themselves are also affected by the higher order sidebands of adjacent meshing harmonics. If the vibration average is band pass filtered about one of the meshing harmonics (normally the largest one), and assuming that the amplitude of that harmonic is much greater than its neighbors, then the interference from the sidebands of the neighboring harmonics will be negligible. The filtered signal can be estimated as [17]:

$$
y_{r}(t)=\left[1+\tilde{a}_{k}(t)\right] X_{k} \cos \left[k N t+\varphi_{k}+\tilde{b}_{k}(t)\right]
$$

Wang [26] showed that, if $\left|\tilde{a}_{k}(t)\right|\left\langle 1,\left|\tilde{b}_{k}(t)\right|\left\langle\left\langle\pi\right.\right.\right.$ and $\left|\tilde{a}_{k}(t) \tilde{b}_{k}(t)\right|\langle\langle 1$, then the residual signal obtained by eliminating the meshing harmonics from the filtered signal and the residual signal is approximated by the following equation:

$$
r_{k}(t)=X_{k} \sqrt{\tilde{a}_{k}^{2}(t)+\tilde{b}_{k}^{2}(t)} \cos \left[k N t+\varphi_{k}-\tan ^{-1}\left(\frac{\tilde{b}_{k}(t)}{\tilde{a}_{k}(t)}\right)\right]
$$

The envelope of this signal is:

$$
e_{k}(t)=X_{k} \sqrt{\tilde{a}_{k}^{2}(t)+\tilde{b}_{k}^{2}(t)}
$$

This is directly related to both amplitude and phase modulation and somehow shows the total modulation strength as a function of time. In other words, it represents the power of the sudden change due to the mating of faulty teeth in a certain frequency.

A summary of the above procedure in the digital domain is as follows:

After transforming the signal to the frequency domain through FFT analysis and estimating the location of the sidebands, which are related to the fault, the outside components of the selected band will be removed by band pass filtering. Then, the meshing harmonics, which are inside the filtered band, are removed by the band-pass and stop-band filtering. At the next step, by inverse FFT the residual signal will be transformed to the time domain and by squaring this signal the power of fault is estimated. The envelope of this signal is the best criterion for the Kurtosis factor estimation. The envelope of the signal should be distributed normally across the whole time axis.

\section{The mathematical basis of the IPS method}

Between various time-frequency distribution methods, the IPS is a kind of "Cohen-class" of "bilinear timefrequency distributions" [4]. One of the important reasons that the IPS is chosen for diagnostic purposes is its less sensitivity to window parameters when compared to other methods. Furthermore, the IPS method can be performed by real-valued signals sampled at the Nyquist rate without having information about the analytical signal [20]. Due to ringing phenomenon, the fault detection of weak signals with the noise is easier. In the "Cohen-class" of time-distribution method, if the Kernel function is chosen as $\phi(\xi, \tau)=\cos (\pi f \tau)$, the result is the Levin's IPS with the following equation [11]: 


\section{CASE FREE}

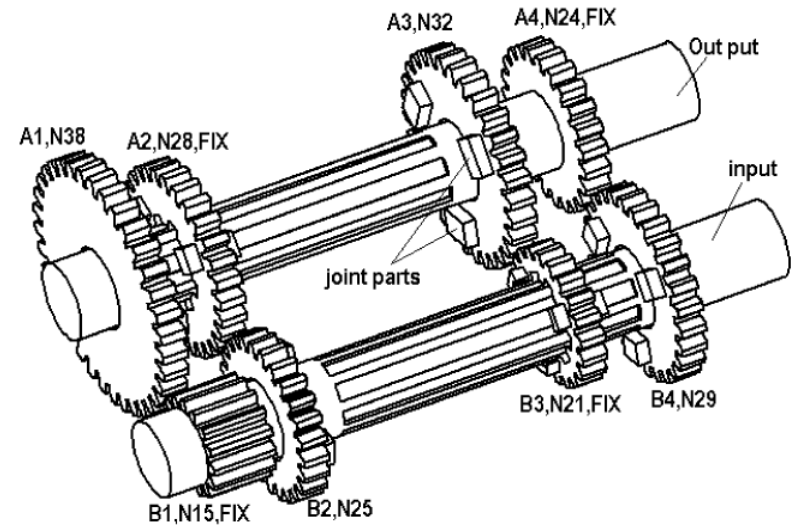

Fig. 1. The position of the gears in the gearbox.

$$
I P S_{x}(t, f)=\frac{1}{2} \int_{-\infty}^{+\infty}\left[x(t) x^{*}(t-\tau)+x^{*}(t) x(t+\tau)\right] e^{-j 2 \pi f \tau} d \tau
$$

Where $\mathrm{x}(\mathrm{t})$ is the time-domain signal and the * sign shows the complex conjugate. The interpretation of the IPS result is simpler than the other methods in the digital signal processing. In other words, the IPS is the FFT of the sum of two autocorrelation functions. In the windowed version of the IPS, the above formula is weighted by some special functions such as different types of window functions. In the frequency domain, if a signal is in the form of:

$$
x(t)=\sum_{i=1}^{n} A_{i} e^{j 2 \pi f t_{i}}
$$

which $A_{i}$ and $f_{i}$ are the amplitude and frequency of signal components, then the IPS of that signal is [27]:

$$
I P S_{x}(t, f)=\sum_{i=1}^{n} \sum_{j=1}^{n} A_{i} A_{j} \delta\left(f-f_{i}\right) \cos \left[2 \pi\left(f_{j}-f_{i}\right) t\right]
$$

When the IPS is studied locally in time events, the signal should be weighted by some kind of weighting functions. Then, the weighted IPS is [19]:

$$
I P S_{y}(t, f)=\frac{1}{2} \int_{-\infty}^{+\infty}\left[y(t) y^{*}(t-\tau)+y^{*}(t) y(t+\tau)\right] e^{-j 2 \pi f \tau} d \tau
$$

For a discrete time signal, the discrete form of the IPS is:

$$
\operatorname{IPS}\left(n, m, \frac{2 \pi}{M}\right)=\sum_{k=L+1}^{L-1}\left[x(n) x^{*}(n-k)+x^{*}(n) x(n+k)\right] w(k) e^{-j 2 \pi k m / M}
$$

where " $n$ " is the time index, " $k$ " is the discrete variable and " $m$ " is the frequency index. " $L$ " is related to the length of the window and has a relationship with M, while M-1 is the last frequency index. Due to simplicity and the presence of the Kaiser window $(w(k))$ in the MATLAB ${ }^{\circledR}$ toolboxes, it is used with the length of $L=150$ and the shape factor of 10 in this paper as a weighting function.

\section{Test rig setup and data acquisition}

The gear box in the test rig is a Yamaha $(125 \mathrm{cc})$ motorcycle gearbox. This is a four stages gearbox, which has one input shaft and one output shaft. A schematic of this gearbox is shown in Fig. 1. Detailed information of each 
Table 1

The gears specification and the stage of engagement

\begin{tabular}{ccc}
\hline Gear & Number of teeth & Stage and speed ratio(S.R.) \\
\hline A1 & 38 & Stage 1, S.R. 0.39 \\
B1 & 15 & \\
A3 & 32 & Stage 2, S.R. 0.62 \\
B3 & 21 & \\
A2 & 28 & Stage 1, S.R. 0.89 \\
B2 & 25 & Stage 1, S.R. 1.2 \\
A4 & 24 & \\
B4 & 29 & \\
\hline
\end{tabular}

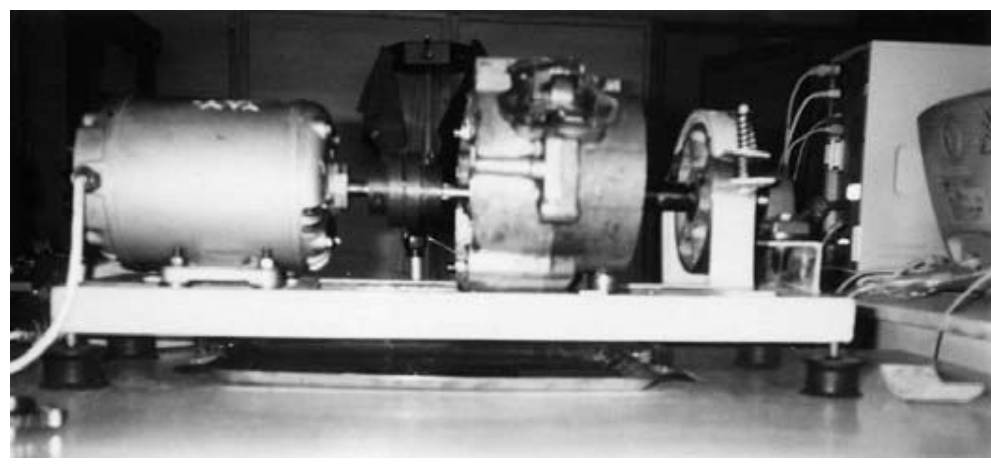

Fig. 2. Test rig overview [3].

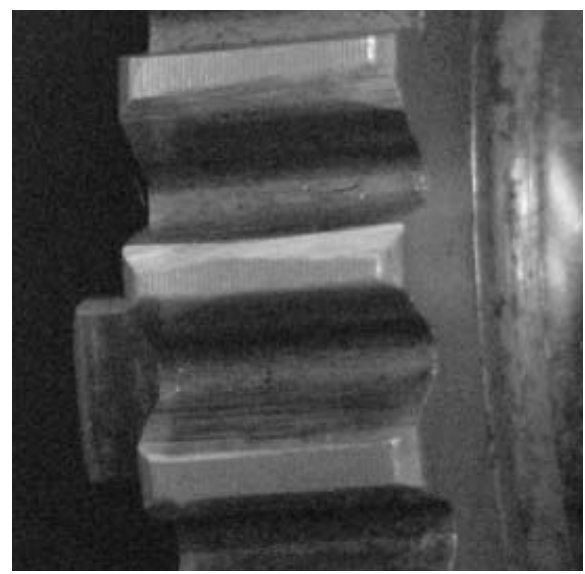

Fig. 3. B4 gear with $5 \%$ fault (5\% eroded tooth).

gear and the speed ratio in each stage is shown in Table 1. The gears, which are under scrutiny in this study, are spur gears with the contact ratio of 1.4 and the pitch module is 2.5 .

A $380 \mathrm{~W}$ electric motor drives the gearbox with the nominal speed of $1420 \mathrm{rpm}$. The connection between driver motor and the gearbox is via a coupling instead of belt connection to avoid additional trembling in the test rig setup. To apply load on the output shaft, a simple mechanism was designed that applies torque on the shaft by providing friction between the pulley and the brake pads. The torque value is controlled by two bolts on the pulley and the springs around the screws, which synchronize the output torque. The test rig with the load mechanism and the driver motor is on a chassis with elastic dampers. The test rig is shown in Fig. 2.

Between the conventional gear faults, two common faults are created on the B4 gear in the 4th stage of the gearbox mating. In this stage, the A4 and B4 gears are mating. By removing 5\% of the tooth crest, the fault is created (Fig. 3). 


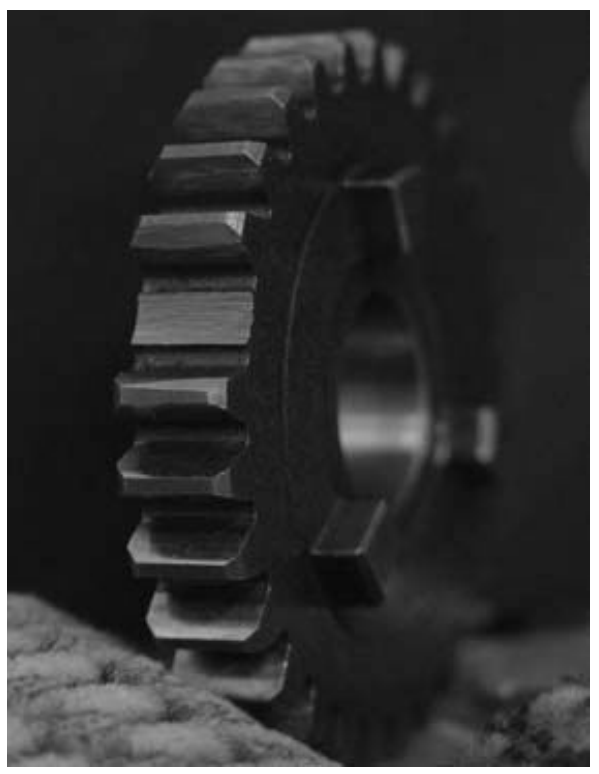

Fig. 4. B4 gear with the broken tooth.

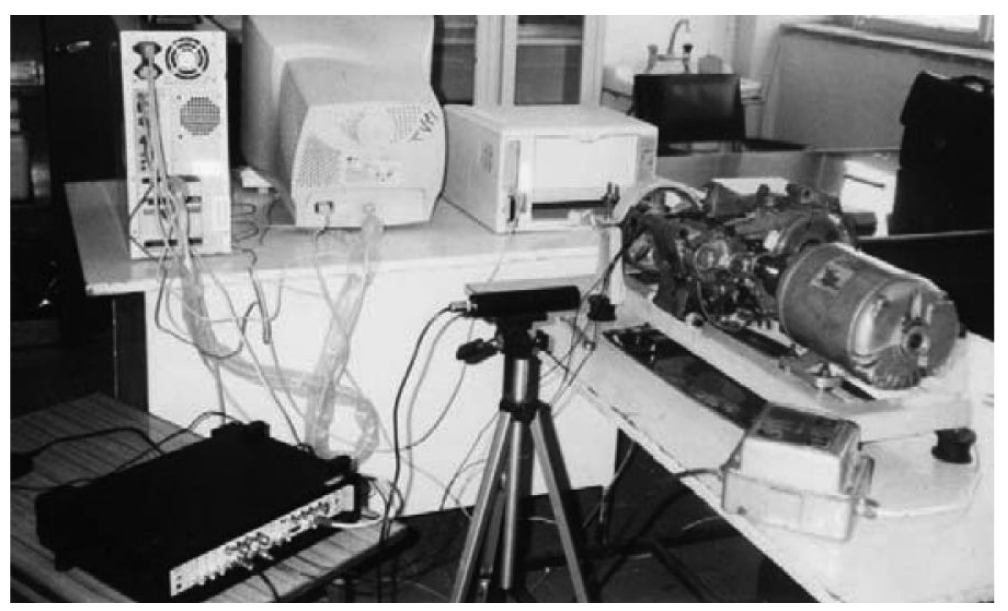

Fig. 5. Test rig and data acquisition system [3].

By increasing the removal process to $20 \%$ of the crest, a $20 \%$ fault is generated. The broken tooth fault is generated by complete removal of that single tooth as it is shown in Fig. 4. It is obvious that the reference signal is acquired before any fault generation.

For data acquisition purposes, a 4 channel "pulse" multi-analyzer and "Pulse labshop" ${ }^{\circledR}$ software is used. Three of the input channels are connected to the $3 \mathrm{D}$ " $\mathrm{B} \& \mathrm{~K}$ " $\circledR$ accelerometer. The accelerometer is mounted on the shaft bearing with the $\mathrm{X}$ direction perpendicular to the shaft. This is due to this fact that the sensitivity of the accelerometer in the $\mathrm{X}$ direction is higher than the other directions. The best location of the accelerometer is found through a trial and error method. For preprocessing, which is time averaging of the raw signal, a tachometer is used in front of the coupling. It is connected to the last channel of the "pulse" data analyzer. A complete view of the test rig and the data acquisition system and instruments is shown in Fig. 5.

At the first stage of data acquisition, the data of the no-fault condition is saved in a PC. The mesh frequency according to the mating gears (A4 and B4) is: $f=\frac{1420}{60} * 29=686.33$. 


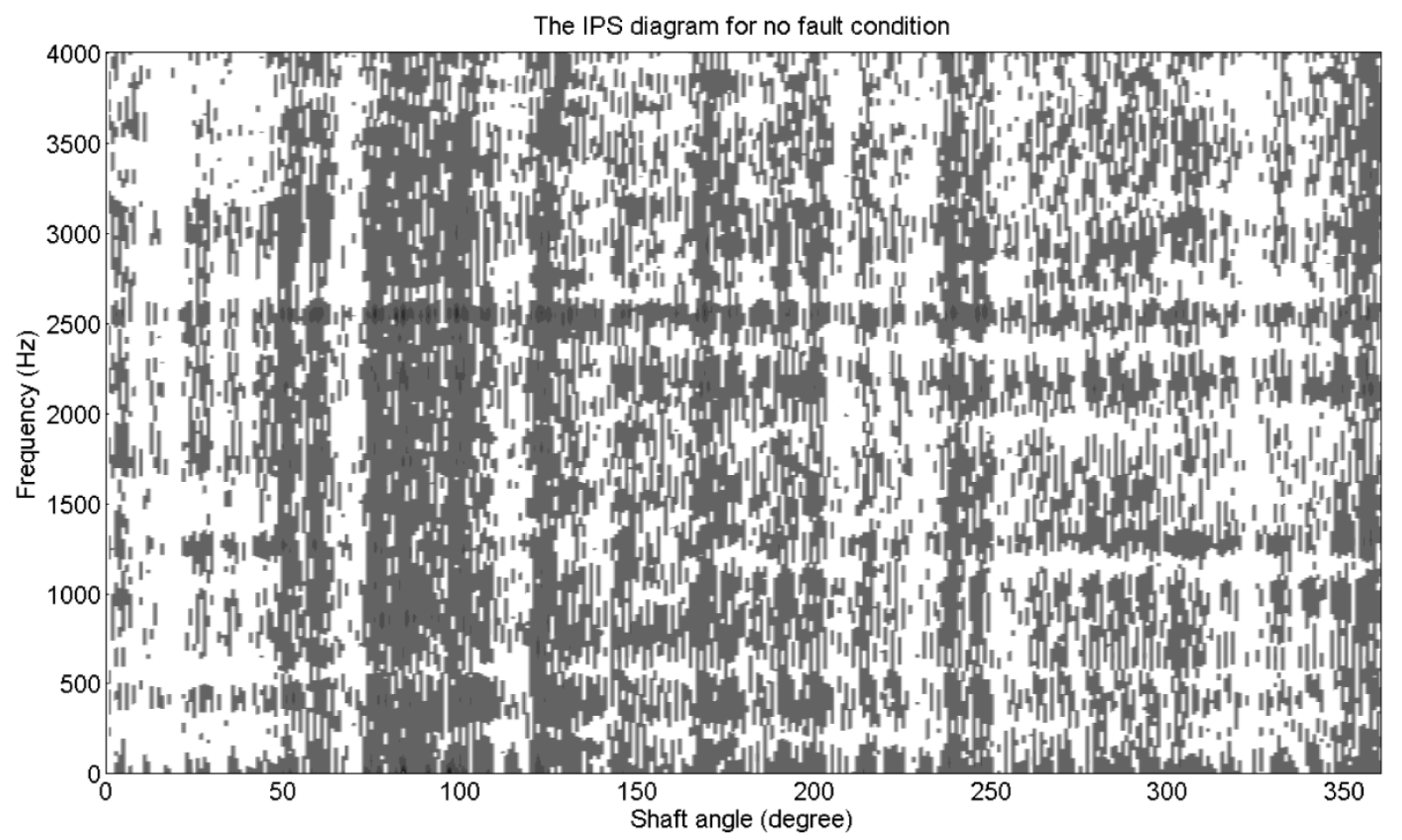

Fig. 6. IPS diagram for no-fault condition.

The high frequency component of the test rig is the 9 th mesh harmonic $(9 * 686.33=6176.97)$. The sampling frequency is chosen to be $16 \mathrm{kHz}$, which is more than twice of the highest frequency component in the test rig. This is in accordance with the Nyquist sampling theorem, in order to avoid the aliasing effect. The averaged vibration signal was stored for 20 complete revolutions. At the second step, the crest of the gear tooth is removed to attain a $5 \%$ fault. As before, the raw data of 20 complete revolutions is stored in a PC. After removing the crest of the gear tooth to investigate the $20 \%$ fault, the same procedure is repeated. At the end, by a complete removal of the tooth, the data for broken tooth is saved. In this way, the averaged vibration signals for four states of no fault, $5 \%$ fault, $20 \%$ fault and the broken teeth are saved. Now, by implementing the codes of these two methods in MATLAB ${ }^{\circledR}$ and uploading the acquired data files, the results of each method are investigated and the comparison and interpretation of the results is performed.

\section{Application of the IPS method to the test rig data}

The application of this method is not as complicated as the resonance demodulation technique. In the IPS method, as a criterion for energy in the time domain, the concentration of energy in specific frequencies (especially at mesh frequency) and at specific time is an indication of the fault presence. In spite of time averaging, the presence of noise confuses the fault detection process in early stages of the fault generation. As with the previous method, the beginning phase of the IPS calculation is the time averaging of raw vibration signals to reduce signal noises. Then, the sum of two autocorrelation functions of the averaged signal is calculated. At the next step, this summation is weighted and normalized by the window function (Kaiser window). At the end, by applying the Fourier transform on the above function, the instantaneous power spectrum (IPS) diagram is obtained. The IPS diagrams of the test rig data for no fault gear (B4), $5 \%$ fault (5\% eroded tooth), $20 \%$ fault (20\% eroded tooth) and the gear with a broken tooth are shown in the Figs 6, 7, 8 and 9 respectively.

It is expected that the energy should concentrate at the gear mesh frequency and its harmonics in a specific angular location. According to the signal noise in the early stages of the fault in the IPS diagram at each frequency (Fig. 7), 
The IPS diagram for $5 \%$ fault condition

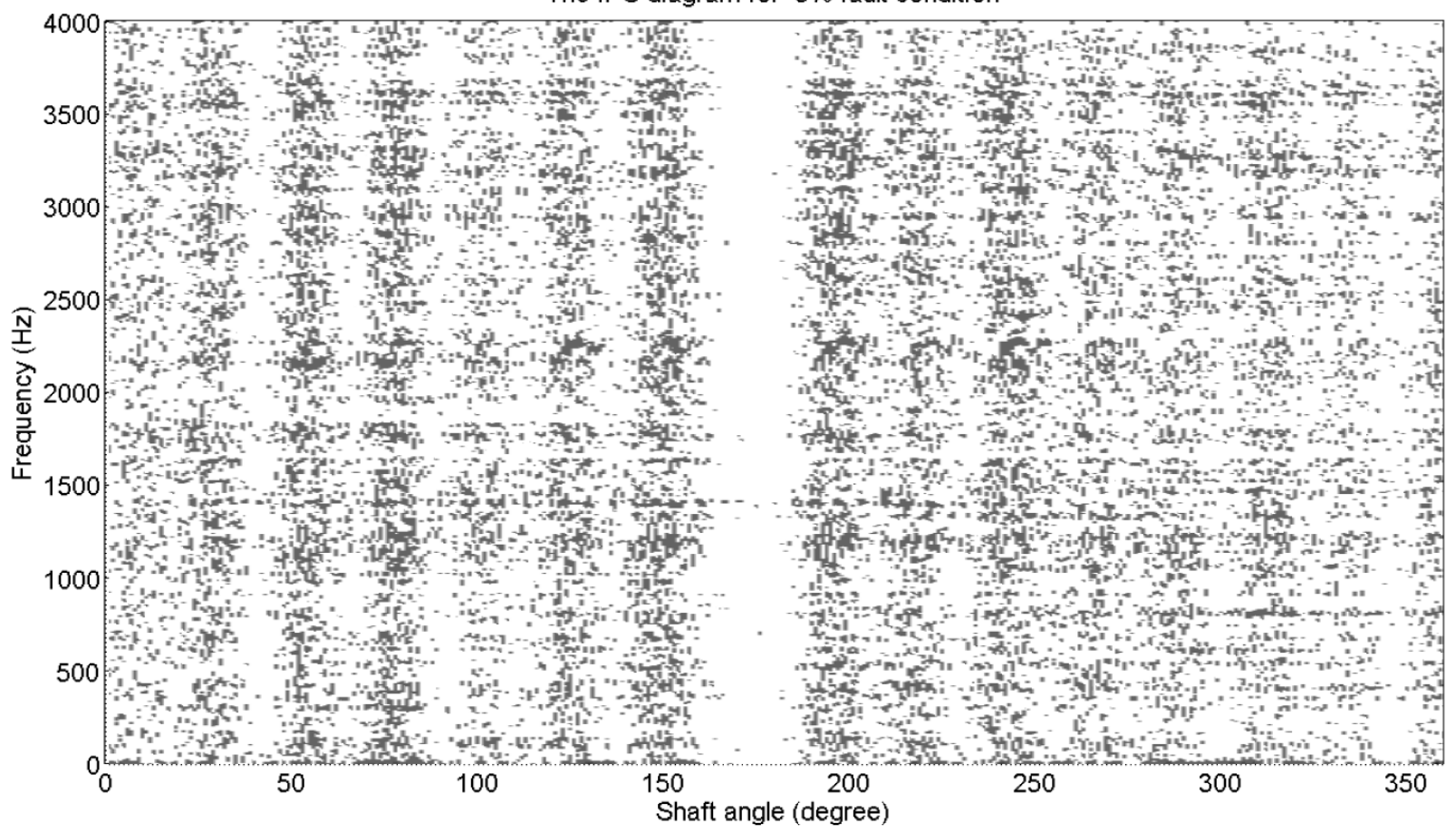

Fig. 7. IPS diagram for $5 \%$ fault condition.

The IPS diagram for $20 \%$ fault condition

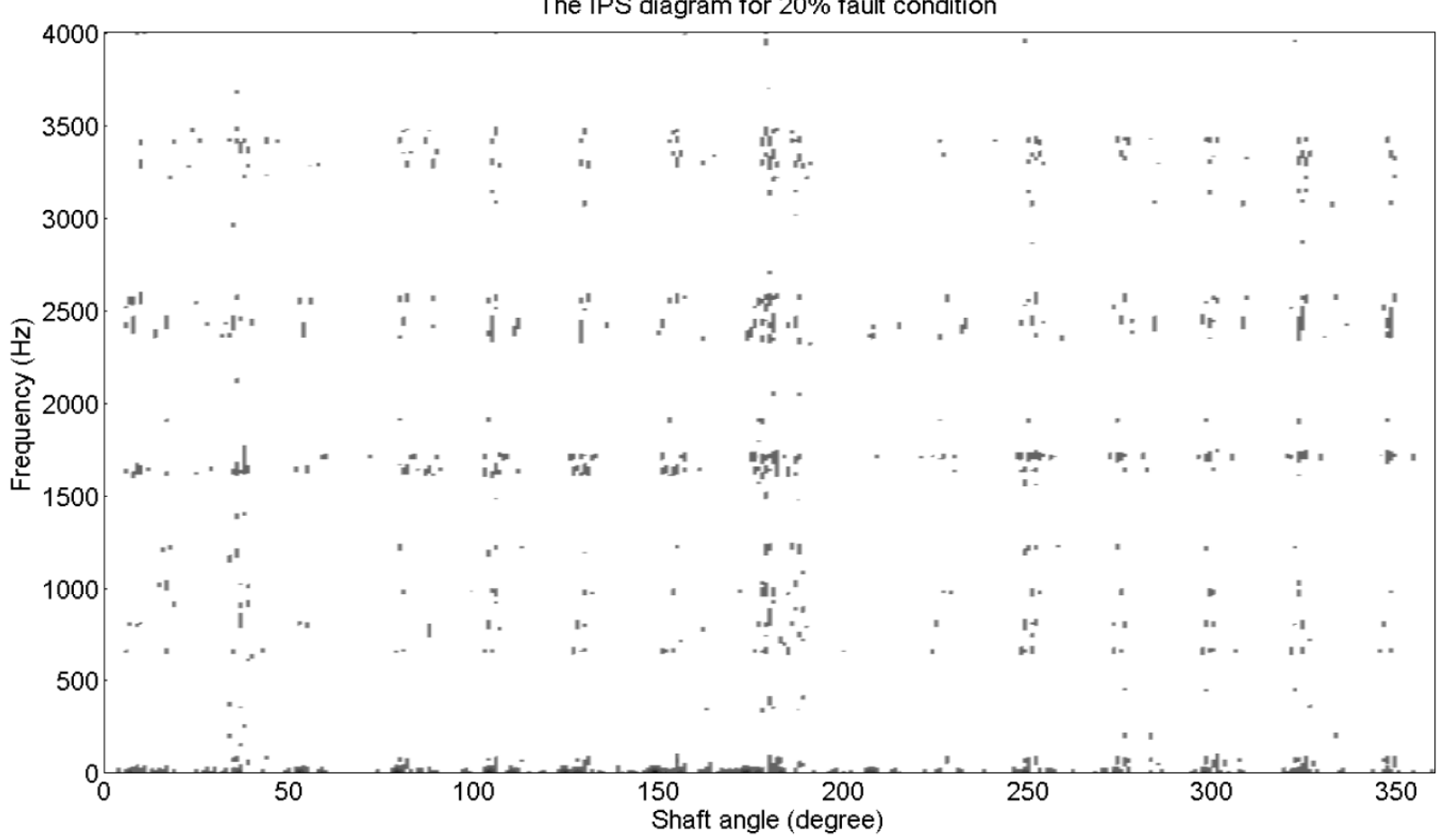

Fig. 8. IPS diagram for $20 \%$ fault condition. 


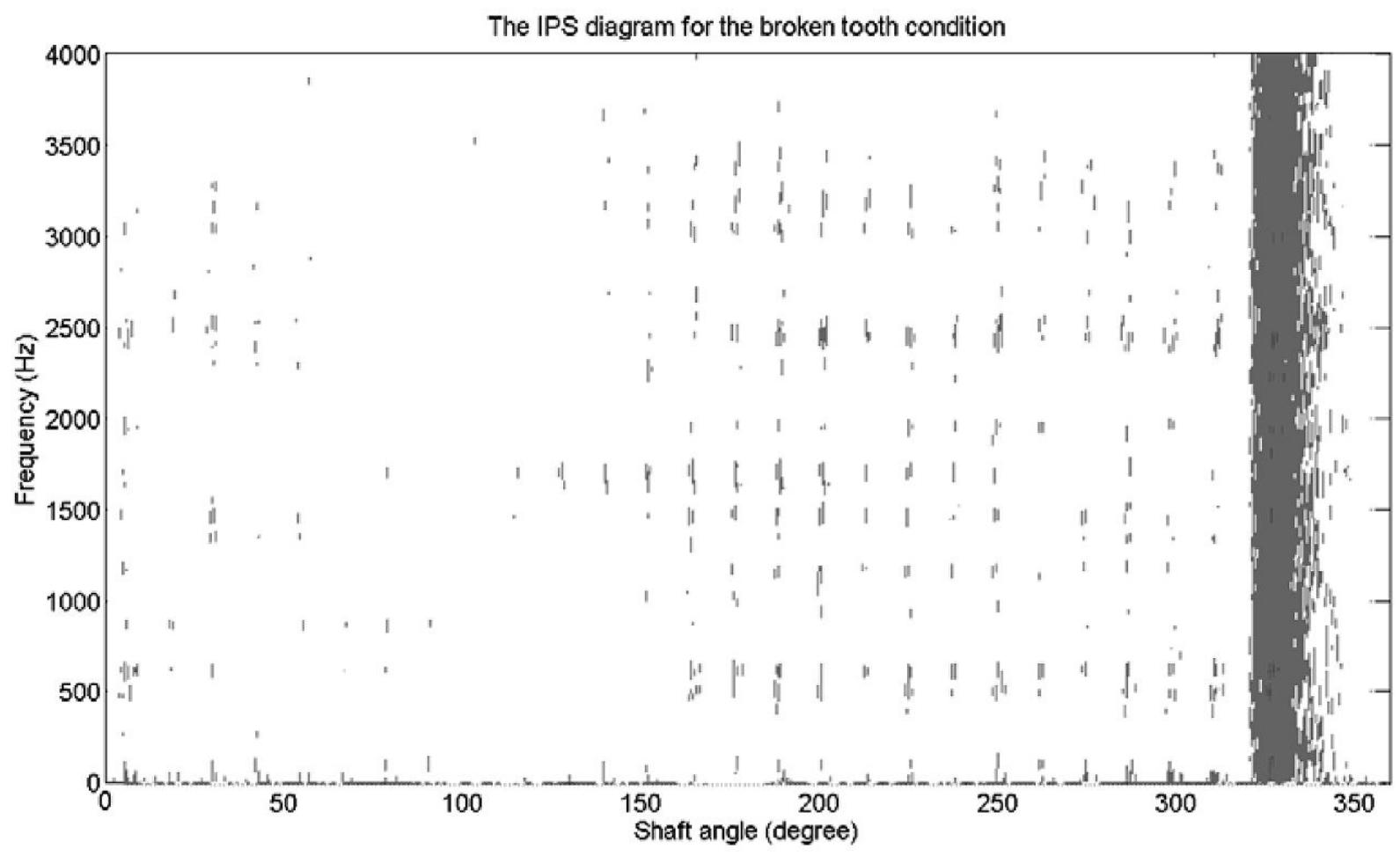

Fig. 9. IPS diagram for fully broken tooth condition.

there is a corresponding point which indicates the noise energy of the noise signal. It is clear that in the no fault condition as in Fig. 6, the noise plays a significant role in the context of the signal with a considerable value of energy. With increasing the fault severity in Figs 8 and 9, it is clear that the energy is going to concentrate more and more in the mesh frequency and its harmonics. The effect of noise is still apparent in the signal context, but is not comparable to the earlier stages of the fault. With increasing the fault severity and as a result, the signal energy, the corresponding noise energy is no longer comparable to energy of the signal generated due to the contact of faulty teeth. In the case of a broken tooth (Fig. 9), around $300^{\circ}$, a high energy modulated spectrum is observed, which occurs at the location of gear teeth mating at the broken tooth. It is important to note that in both of the resonance demodulation and the IPS methods (the Results of the resonance demodulation method will be shown later in the succeeding section), the location of fault at the broken tooth case is around $300^{\circ}$, which verifies the algorithms and computations of both methods.

\section{Application of the resonance demodulation technique to the test rig data}

In this stage, by applying the time averaging method to the raw data, one may produce a representative cycle of the gear vibration signal for each case of fault. Then, by using the Fast Fourier Transform (FFT), the signal is transformed to the frequency domain. The peaks of the modulated signal, accompanied with the sidebands in the spectrum at the mesh frequency and its harmonics, are visible. The sidebands around the first peak of the modulated signal are due to the leakage of the signal, which are appearing as a result of geometrical errors of gear and the weakness of data acquisition systems [15]. In the resonance demodulation technique, the fault appears as sidebands around the higher order mesh frequency harmonics. Therefore, by simple observation of the frequency spectrum of the signal, these sidebands are first detected and removed by filtering (band pass filtering) in the frequency domain and then the resonance demodulation technique is applied on the filtered part of the signal.

As a case study, the above procedure is explained for a no fault case and for the other cases (5\%,20\% and removed tooth) only the results will be shown. In Fig. 10, a representative time averaged signal for no fault case versus Shaft 
angular rotation is shown. It is clear that no feature of the signal can be estimated in this form. By applying FFT, the frequency spectrum of the signal is attained and shown in Fig. 11. The 4th mesh harmonic occurs at around $2760 \mathrm{~Hz}$ and the next harmonics are at $3500,4140,4830 \mathrm{~Hz}$. As it was mentioned above, the preferred sidebands are those that are not around the initial peaks. With a short glance, a side band around the peak at 7 th mesh harmonic is apparent. With the use of "Chebyshev" (type II) filter, which is programmed in MATLAB ${ }^{\circledR}$, by double filtering (first band pass filtering and then band stop filtering) the sideband is extracted from the signal, which is called the residual signal. This residual part of the signal is shown in the frequency domain $(4000-5000 \mathrm{~Hz})$ in Fig. 12 and in the time domain in Fig.13. Then, the residual signal is squared. The envelope of the squared residual signal is shown in Fig. 14. The Kurtosis factor for this kind of squared signal is determined as 3.5. This value of Kurtosis factor does not indicate any fault.

The above procedure is repeated for the case of 5\% fault (5\% eroded tooth). The frequency spectrum of this case is shown in Fig. 15. In the 4th and 7th harmonics, peaks are surrounded by the side bands. The squared envelope of these two residual signals for these two sidebands is shown in Figs 16 and 17 respectively. The Kurtosis factor for the 4 th harmonic is 4 and for the 7 th harmonic is 32 . The Kurtosis value of 32 indicates the presence of a fault in 7th harmonic around $100^{\circ}$ and the Kurtosis value of 4 indicates no fault in that mesh harmonic. As shown in the Fig. 18, the demodulated phase around $100^{\circ}$ has a lag value of $\pi$, which indicates the presence of a fault.

In the case of $20 \%$ fault (20\% eroded tooth), a peak surrounded by the sidebands in the frequency spectrum of the signal (Fig. 19) is visible around $4800 \mathrm{~Hz}$ (7th harmonic). By extracting the residual signal and squaring it, the Kurtosis value is determined as 25. The squared envelope of the residual signal is shown in Fig. 20. The Kurtosis value of 25 indicates the fault and decreasing the Kurtosis value with respect to the $5 \%$ fault case indicates the progression of the fault. The fault is apparent at around $80^{\circ}$ in the envelope curve and in the demodulated phase diagram, it is apparent that around $80^{\circ}$ a phase lag of $\pi$ has occurred (Fig. 21).

In the broken tooth case, two peaks with sidebands appear in the frequency spectrum of the representative signal. The first peak is between the 4th and the 5th harmonics and the other one is at the 9th harmonic at $6000 \mathrm{~Hz}$ (Fig. 22). As the previous cases, the squared envelope of the residual signal is obtained for the signal between 4th and 5th harmonics in Fig. 24 and for the 9th harmonic in Fig. 25. It is obvious that at $300^{\circ}$ (around the 9th harmonic) there is a sever fault (according to the decrease in Kurtosis value with respect to the last case).

By referring to the time averaged raw vibration signal in Fig. 23, it is visible that a large peak exists around $300^{\circ}$. This certifies the fact that the resonance demodulation method exactly estimates the location of the fault and the demodulated phase diagram indicates a phase lag of $\pi$ at about $300^{\circ}$ (Fig. 26).

Therefore, the conclusion remarks that by using the resonance demodulation technique for any case of fault severity, the fault and its location can be estimated and by an increment in the fault severity, the Kurtosis factor tends to be decreased.

\section{Conclusions}

In the resonance demodulation technique, gearbox fault diagnosis, according to Kurtosis factor for the residual signal, is easy. At the first step of fault generation, the Kurtosis factor has a large value. Gradually, with increasing of the fault severity, this factor reduces. Even in this case, the prediction of fault is accomplishable and the Kurtosis factor is still greater than the no fault case. Thus, with the resonance demodulation technique, the prediction of fault in each case of fault severity is possible. The angular location of the fault can also be estimated in the squared envelope curve of the residual signal. The noise has no interference in calculations, which is one of the major advantages of this method.

In the IPS method, noise signal (due to leakage, inappropriate test rig positioning, etc.) interferes with the interpretation of the results, especially in the early stages of fault generation. It is due to the fact that the noise signals produces energy at each frequency, which is mixed with the signal energy in the IPS method. Therefore, the inability of fault detection in the early stages of fault is the drawback of this method. This can be improved by choosing a more complicated time averaging technique such as interpolation according to the speed variation [23]. Gradually, with increasing the fault severity, the energy of the noise in the IPS diagram tends to be insignificant with respect to the IPS levels at mesh harmonics. The location of the fault can be predicted at high level fault severities. Consequently, the IPS is a powerful method for fault detection at higher level fault severities. 


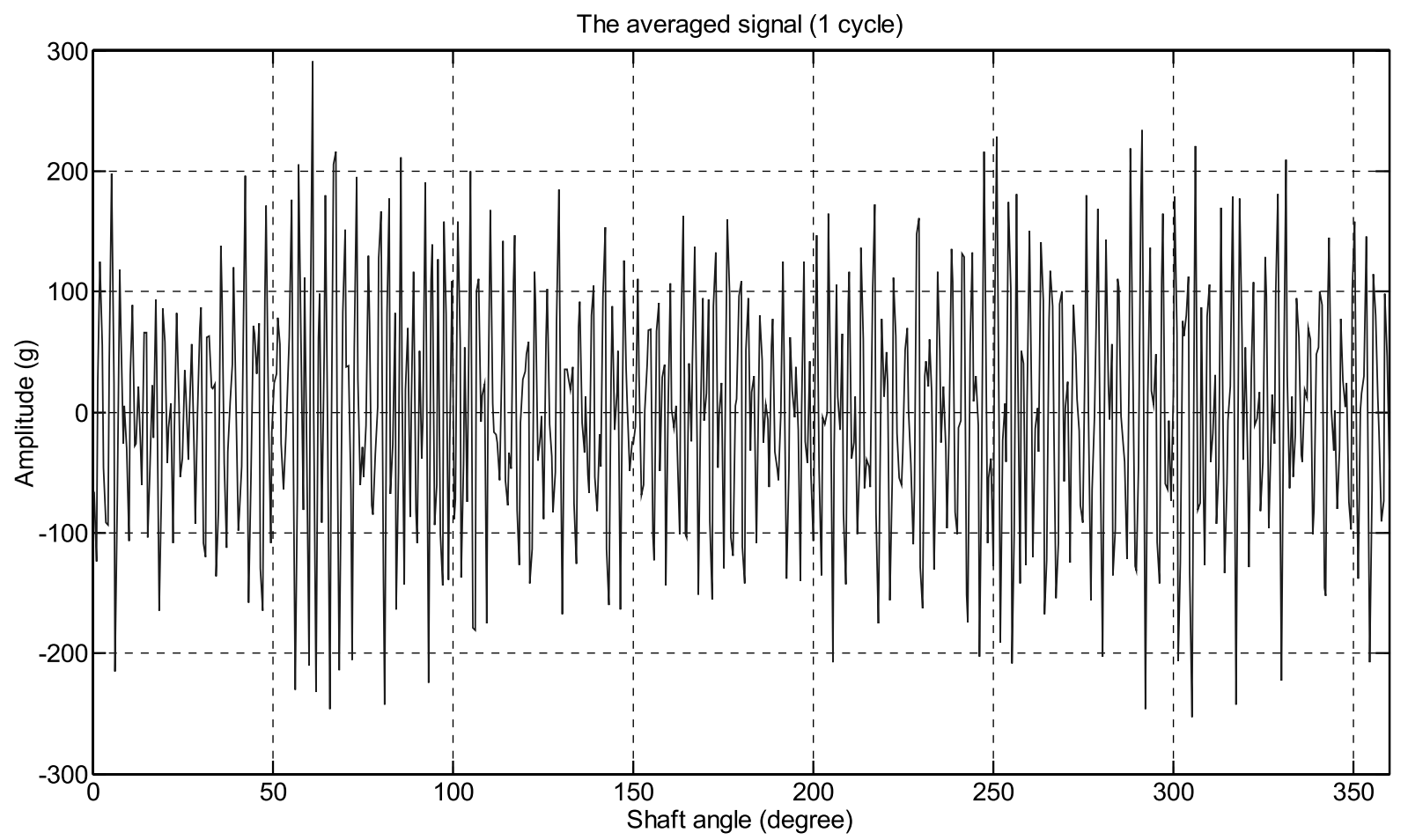

Fig. 10. Time averaged vibration signal for no-fault gear.

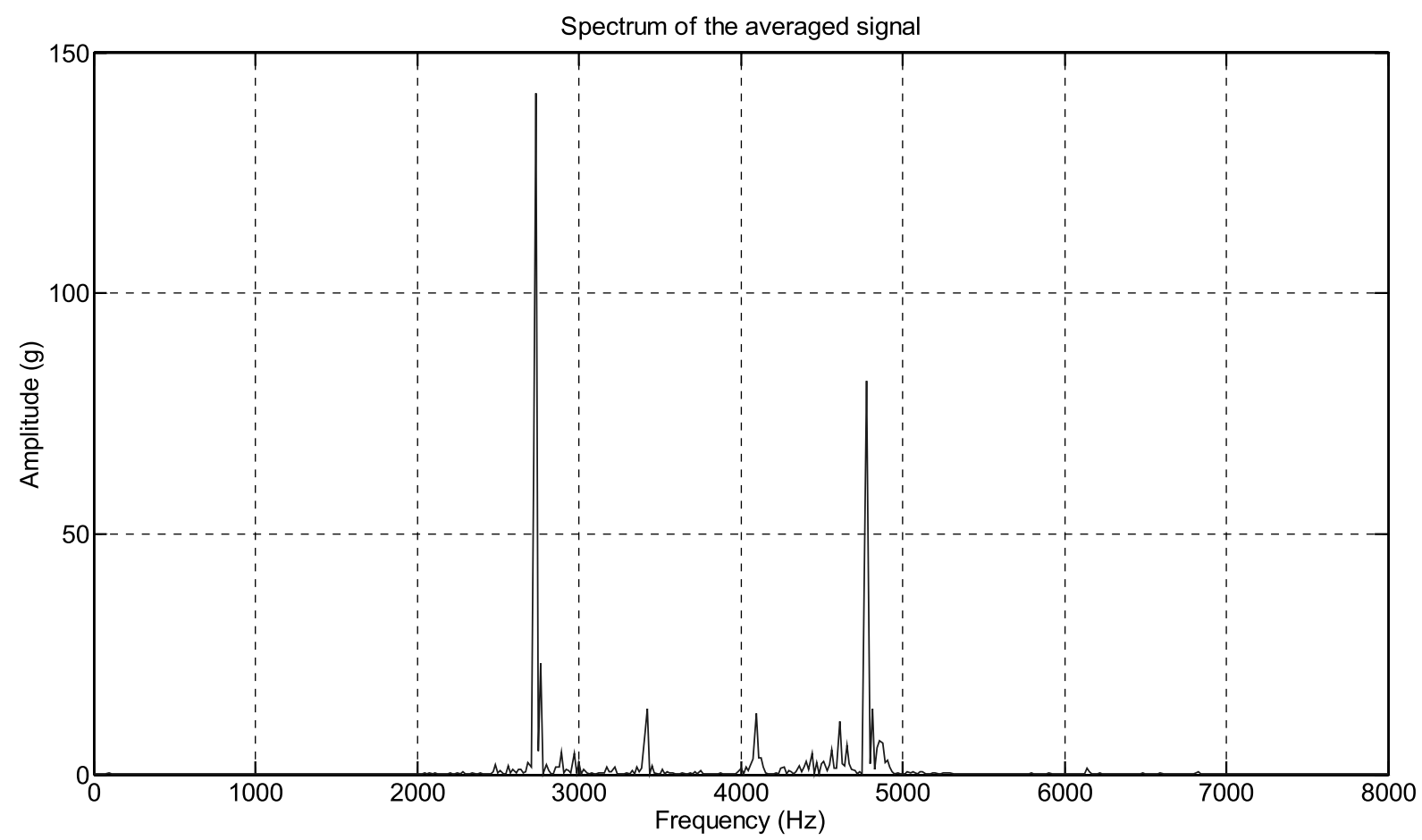

Fig. 11. Frequency spectrum of the time averaged vibration signal. 


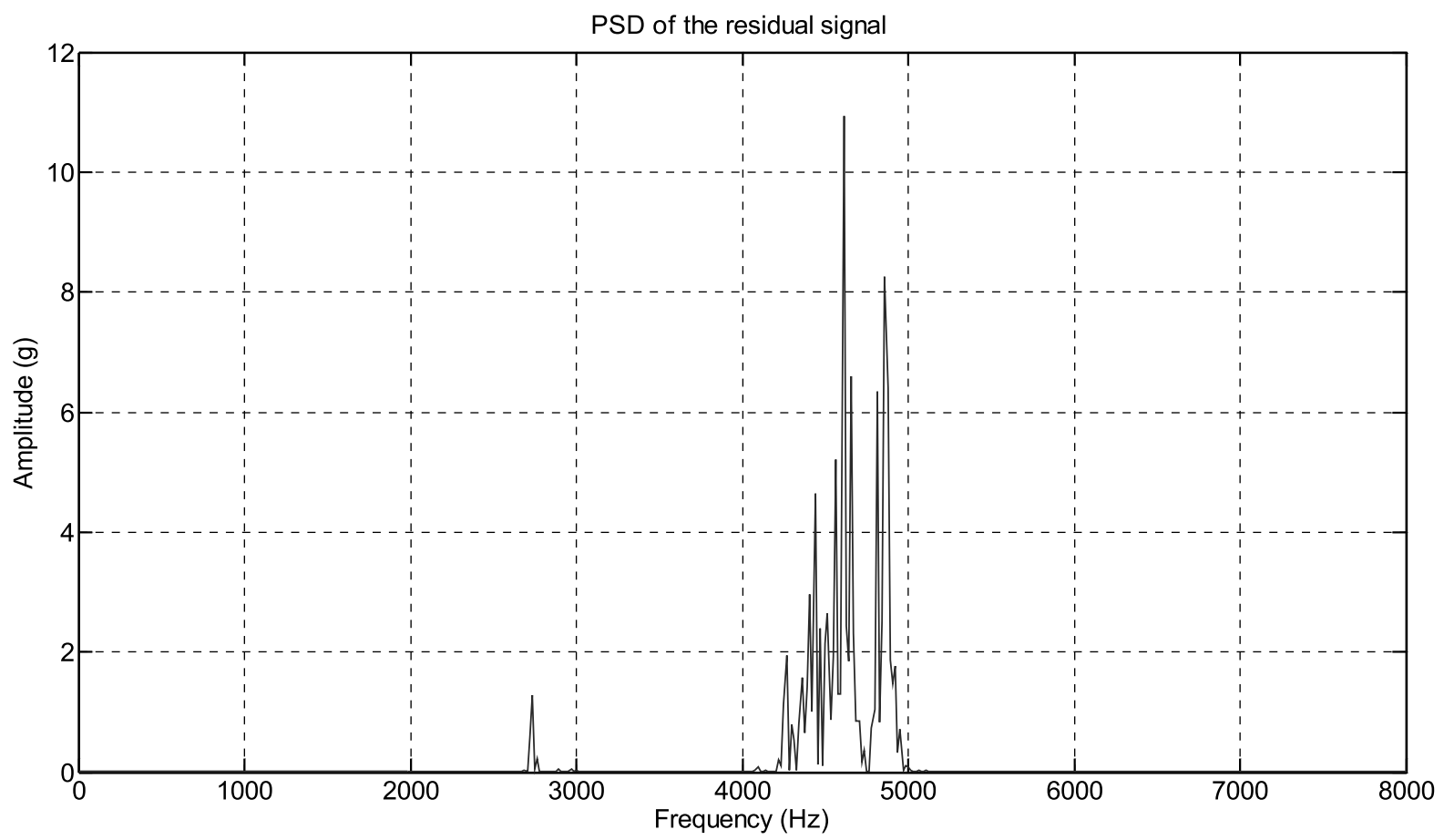

Fig. 12. Residual signal in the frequency domain.

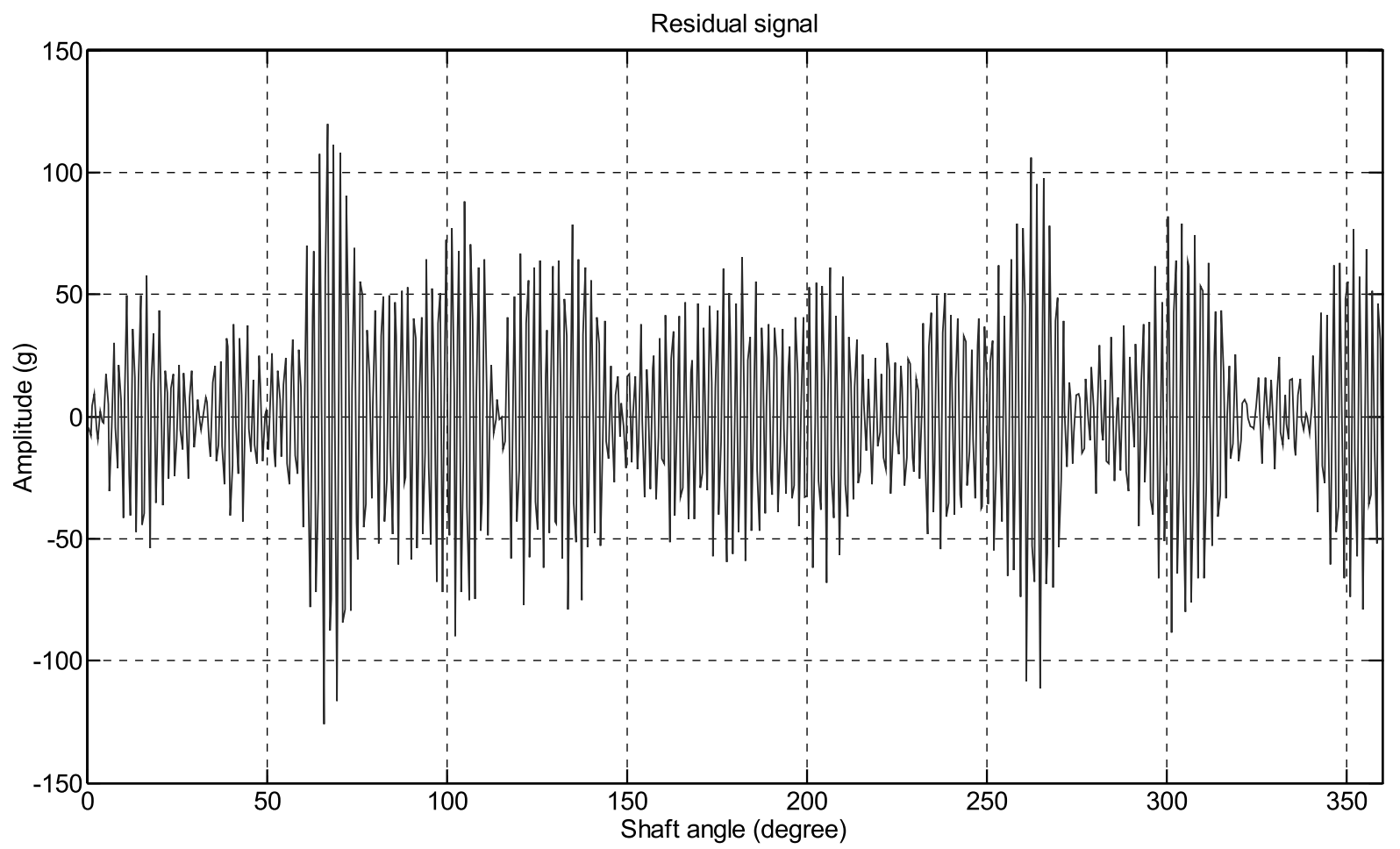

Fig. 13. Residual signal in the time domain. 


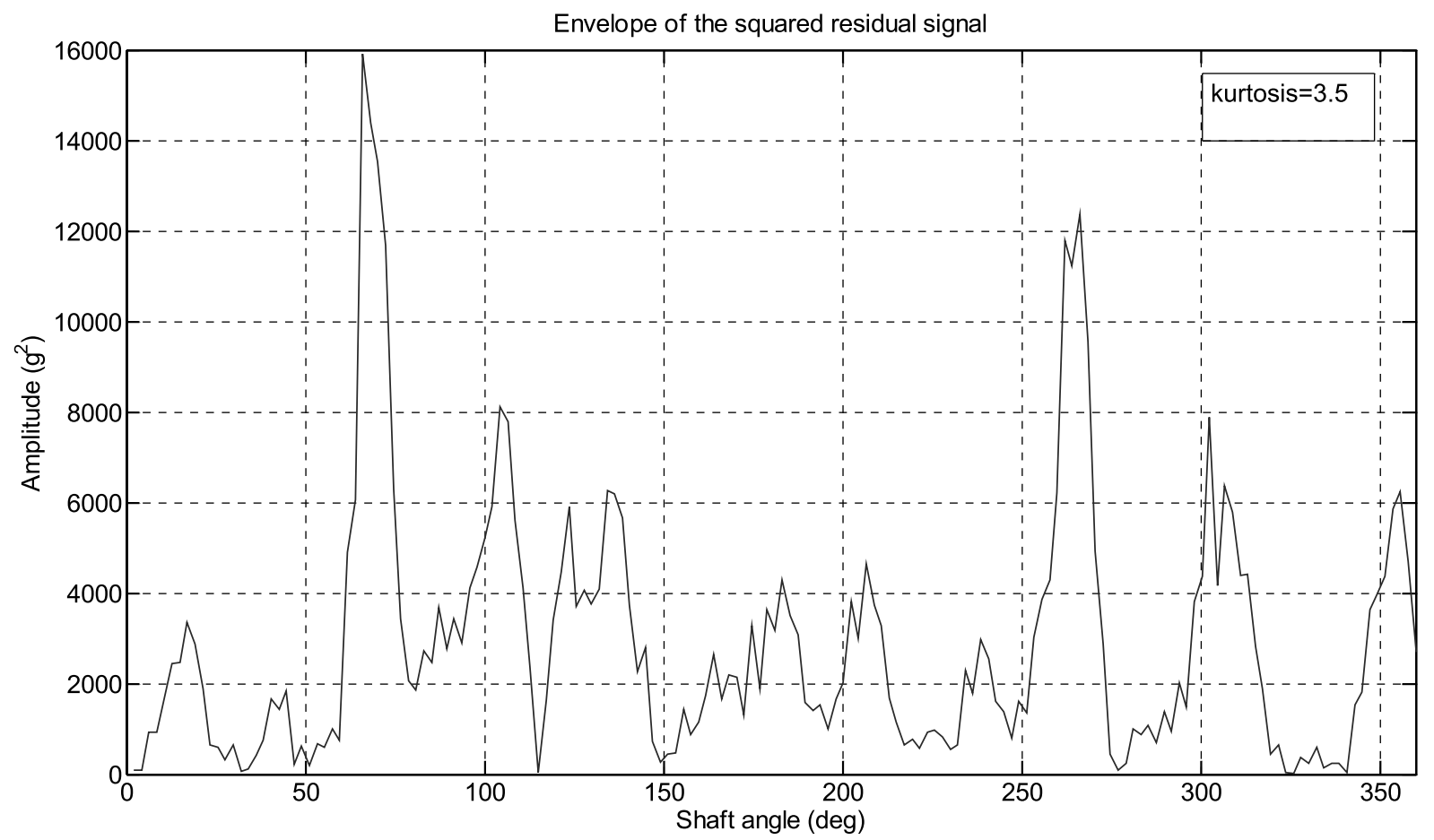

Fig. 14. Envelope of the squared residual signal in the time domain.

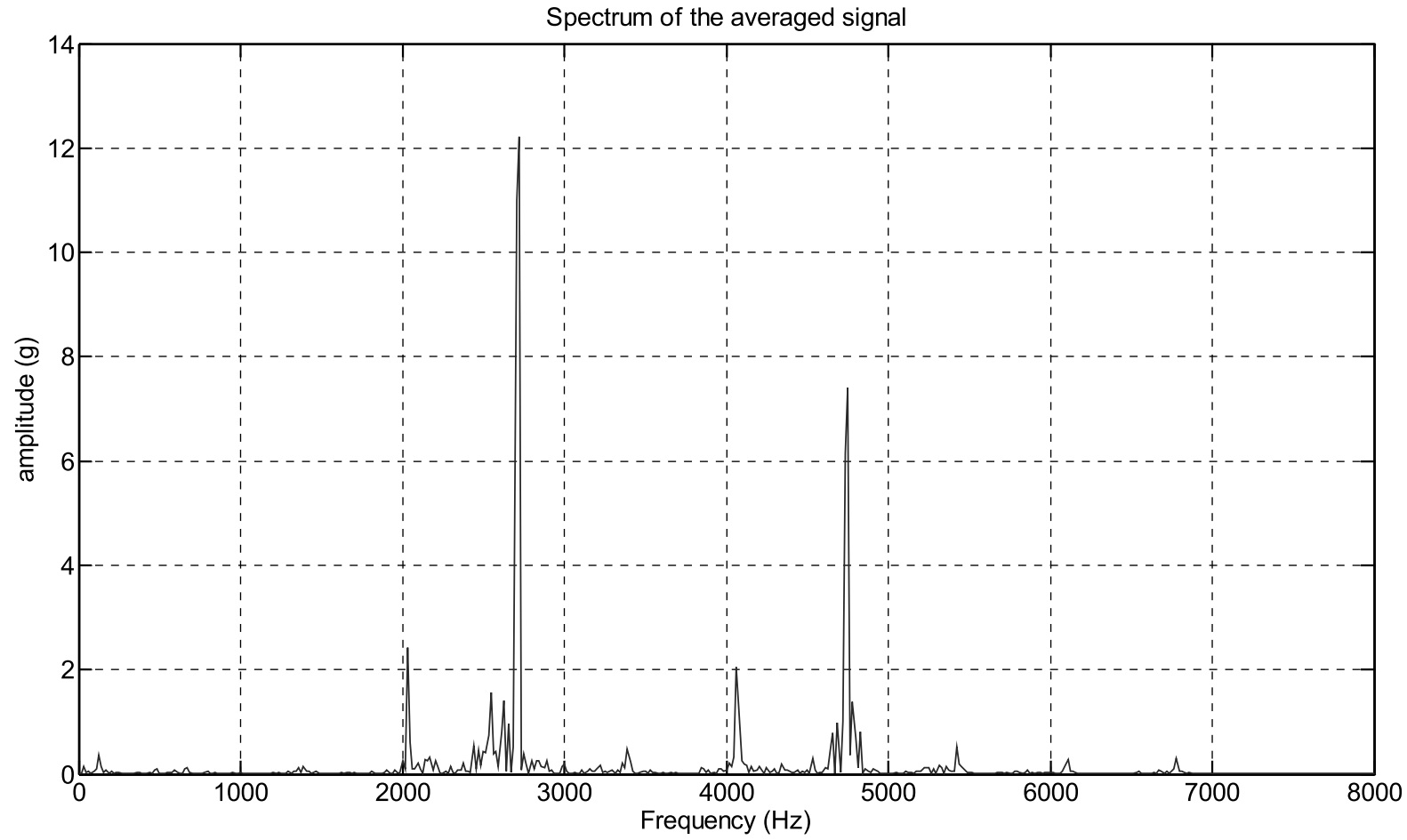

Fig. 15. Frequency spectrum for the $5 \%$ fault. 


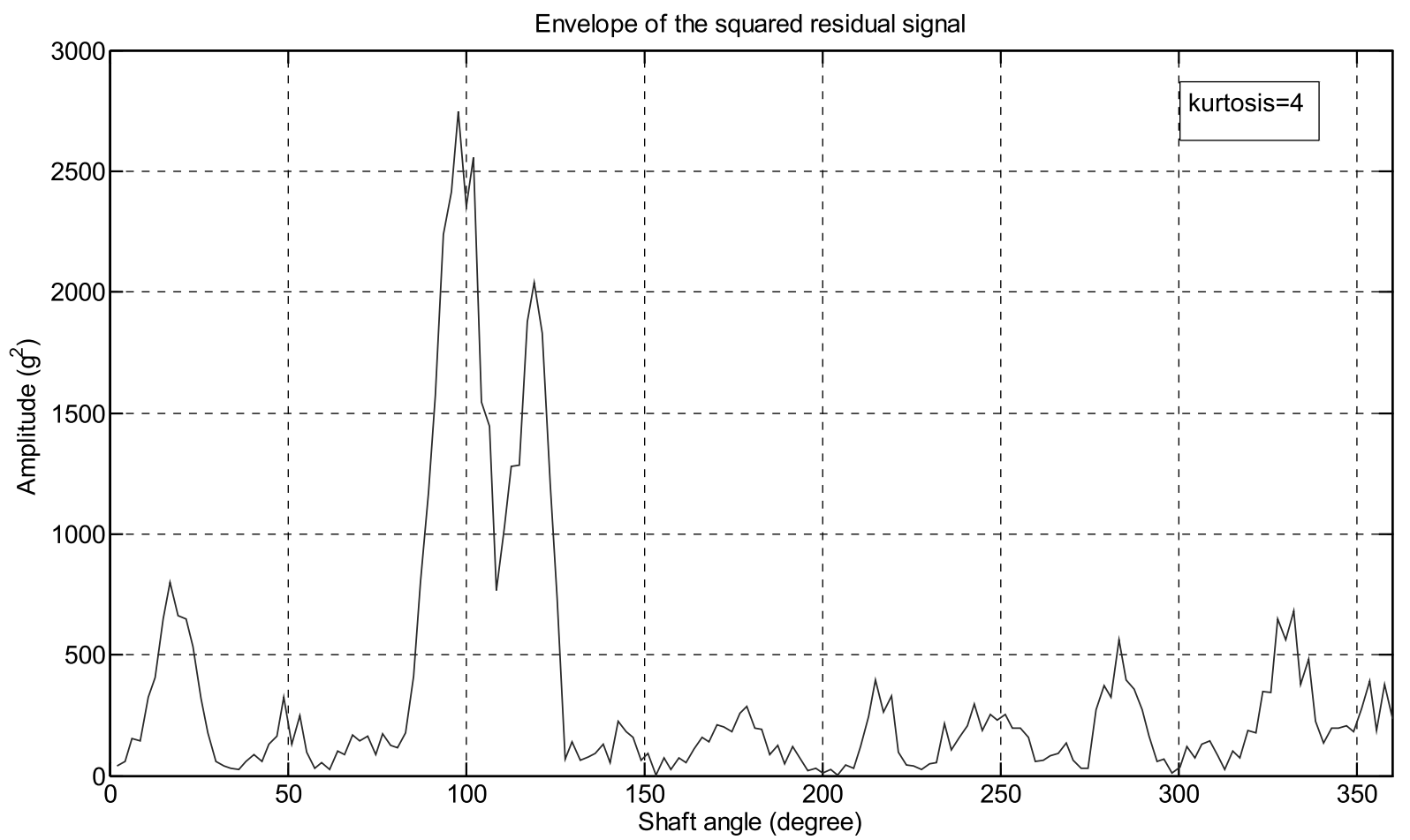

Fig. 16. Envelope of the squared residual signal in the time domain for 5\% fault in the 4th harmonic.

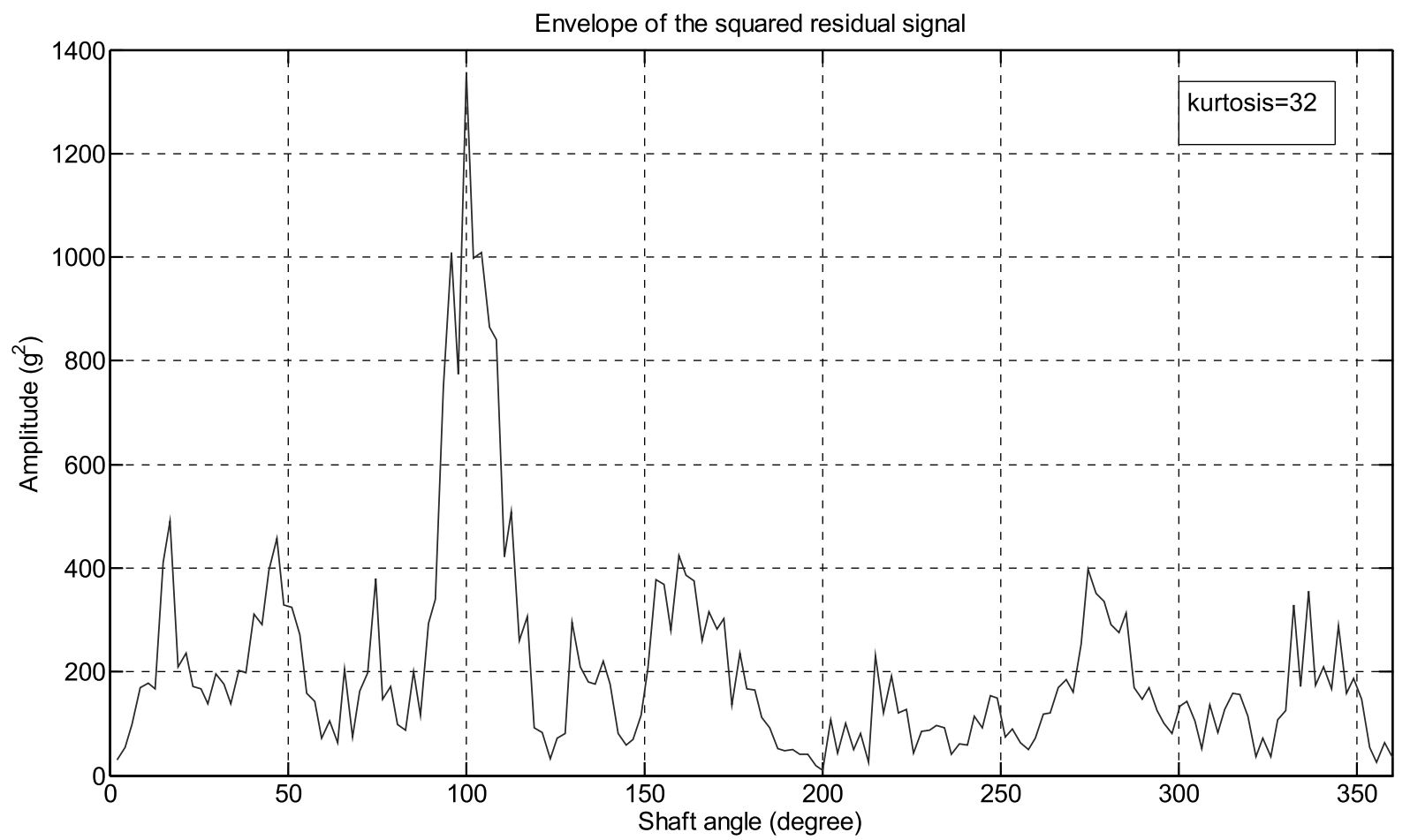

Fig. 17. Envelope of the squared residual signal in the time domain for 5\% fault in the 7 th harmonic. 


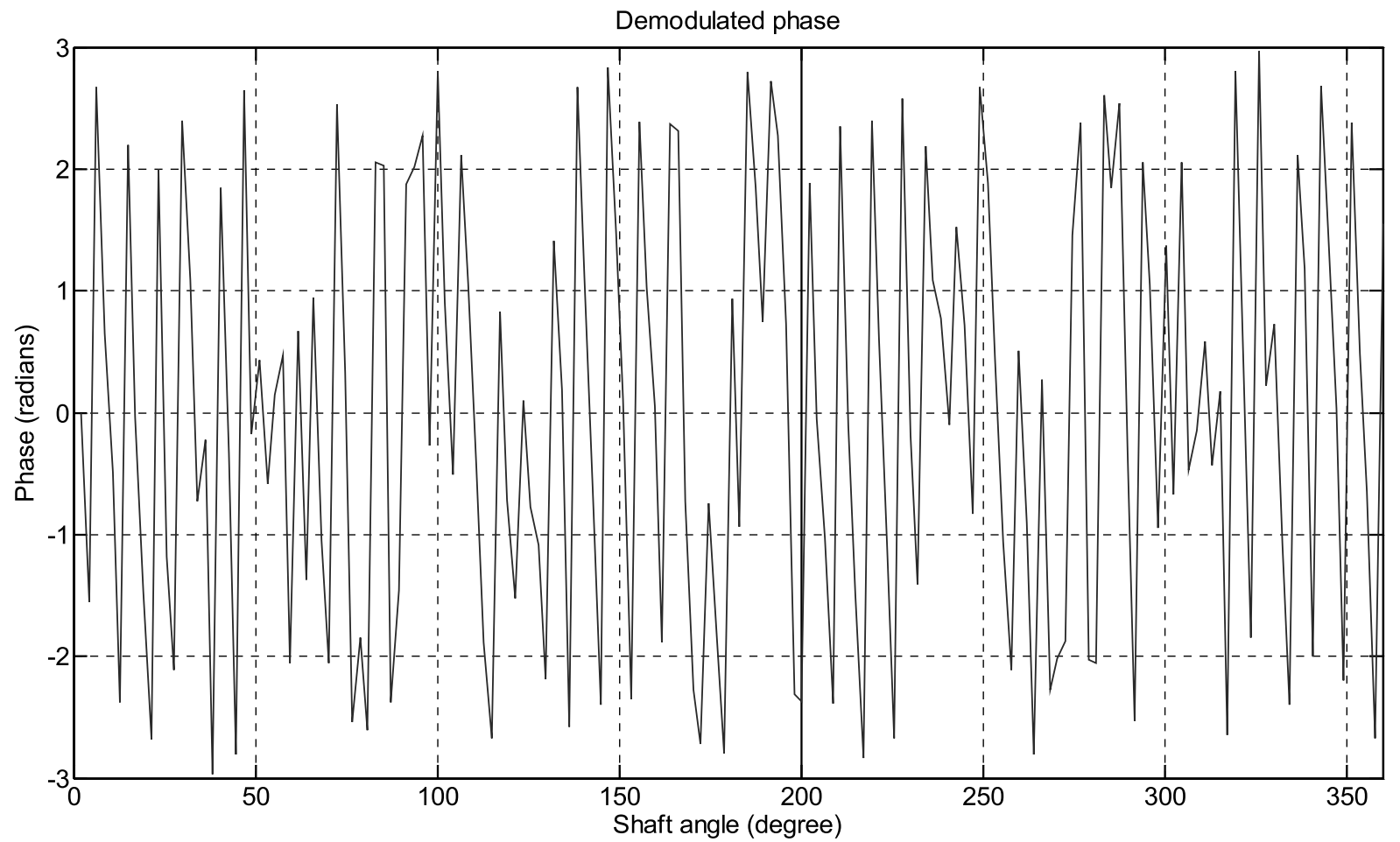

Fig. 18. Phase diagram of the residual signal in the 7 th harmonic for $5 \%$ fault.

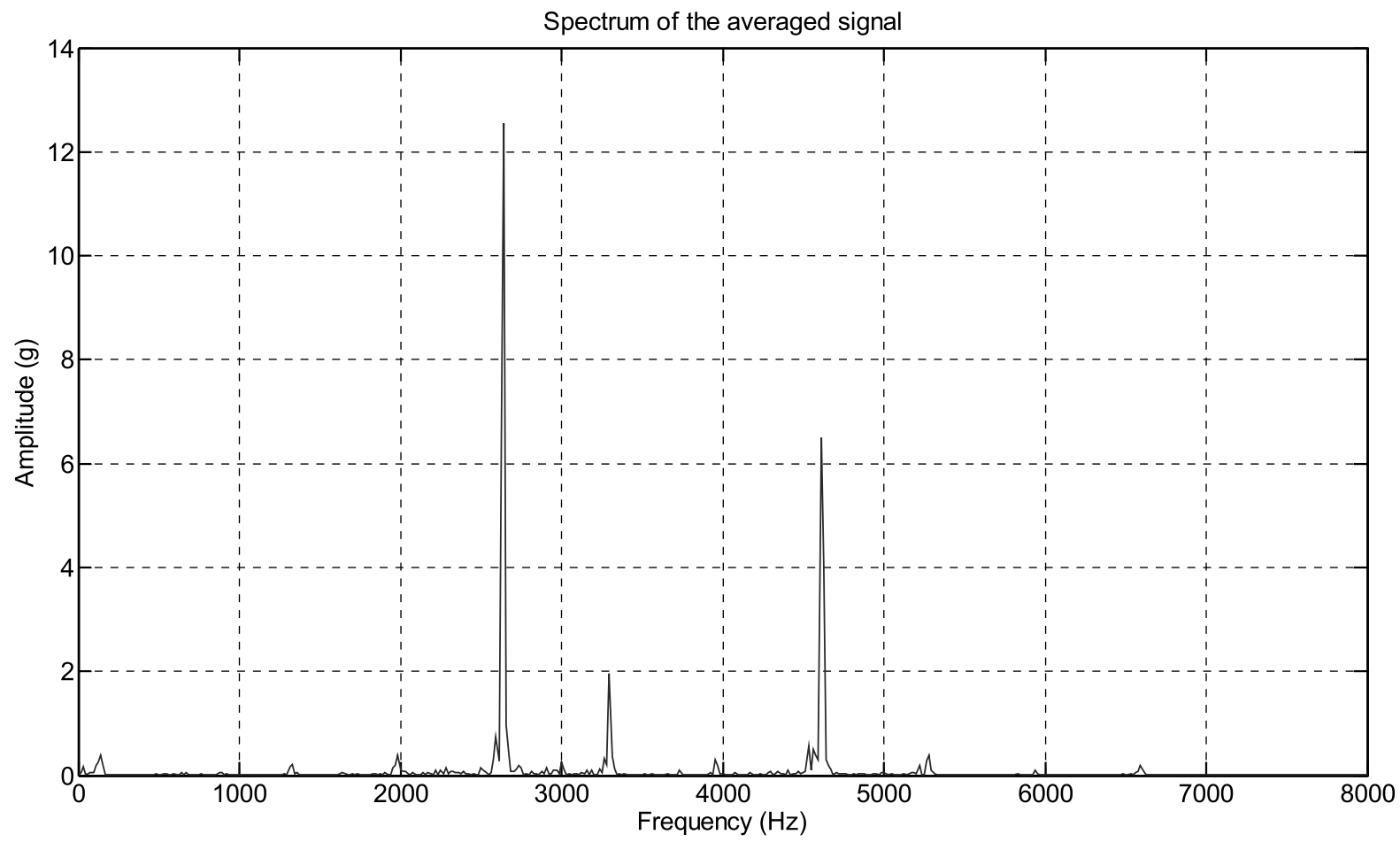

Fig. 19. Frequency spectrum for the $20 \%$ fault.. 


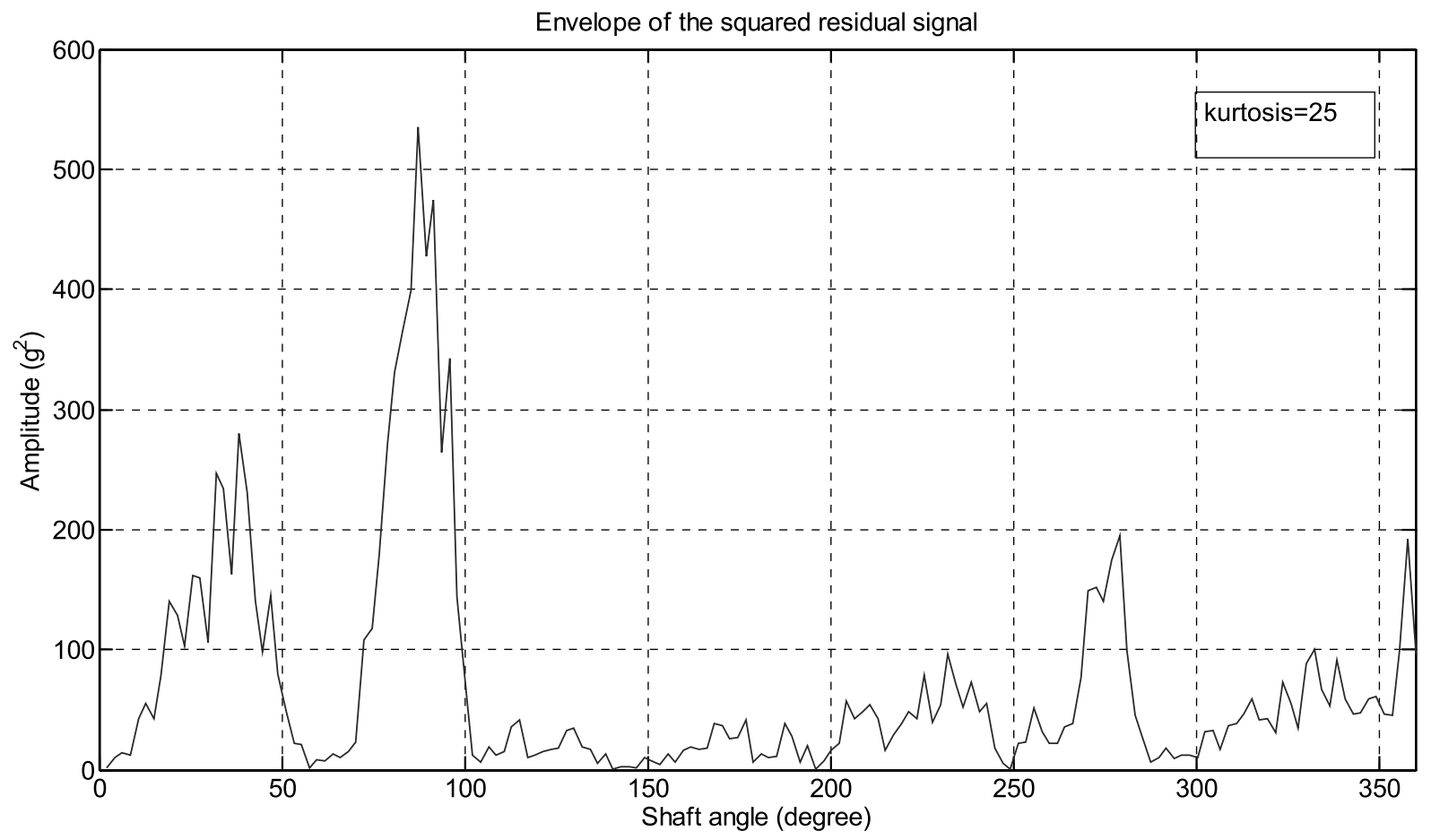

Fig. 20. Envelope of the squared residual signal in the time domain for $20 \%$ fault in the 7 th harmonic.

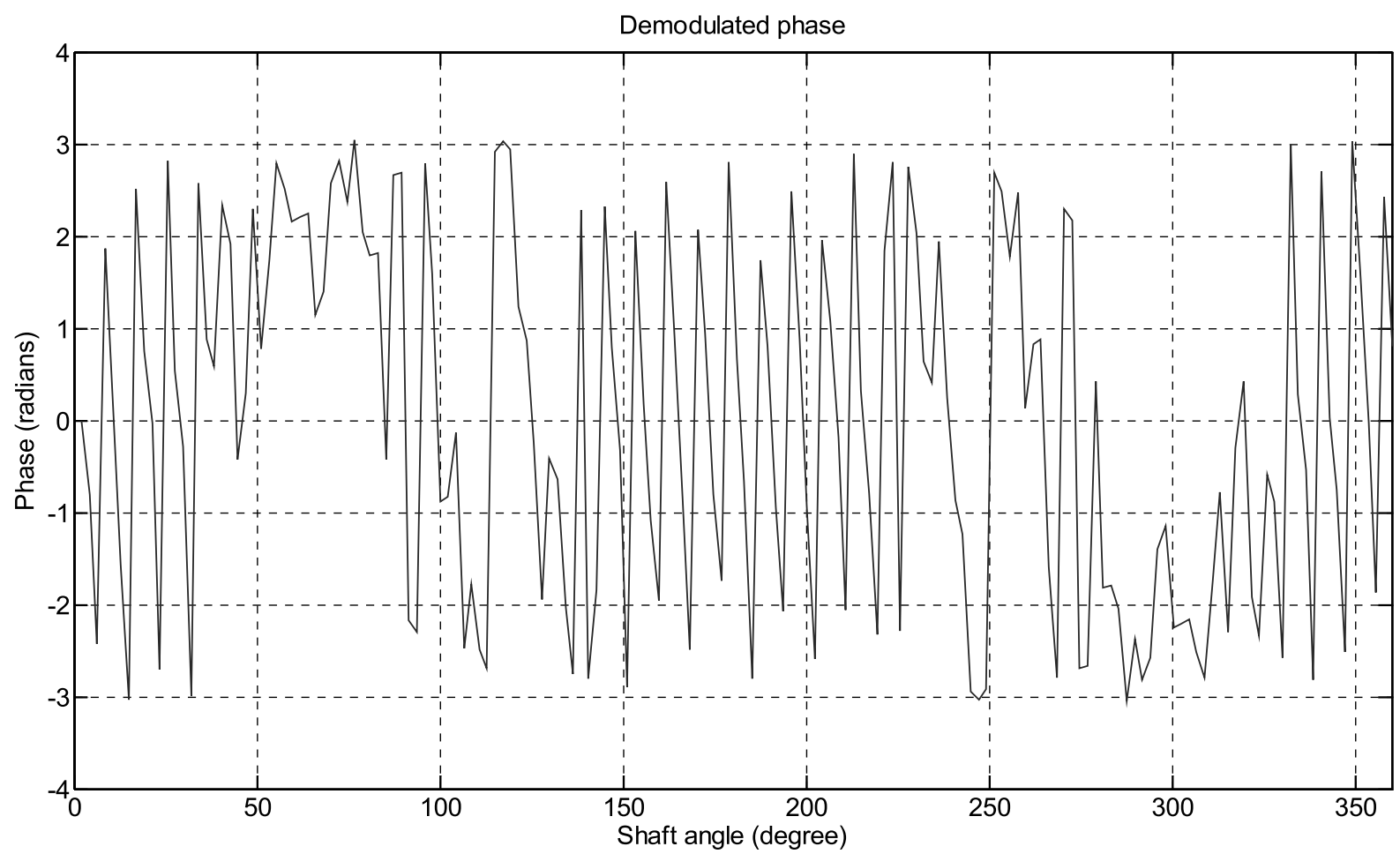

Fig. 21. Phase diagram of the residual signal of $20 \%$ fault. 


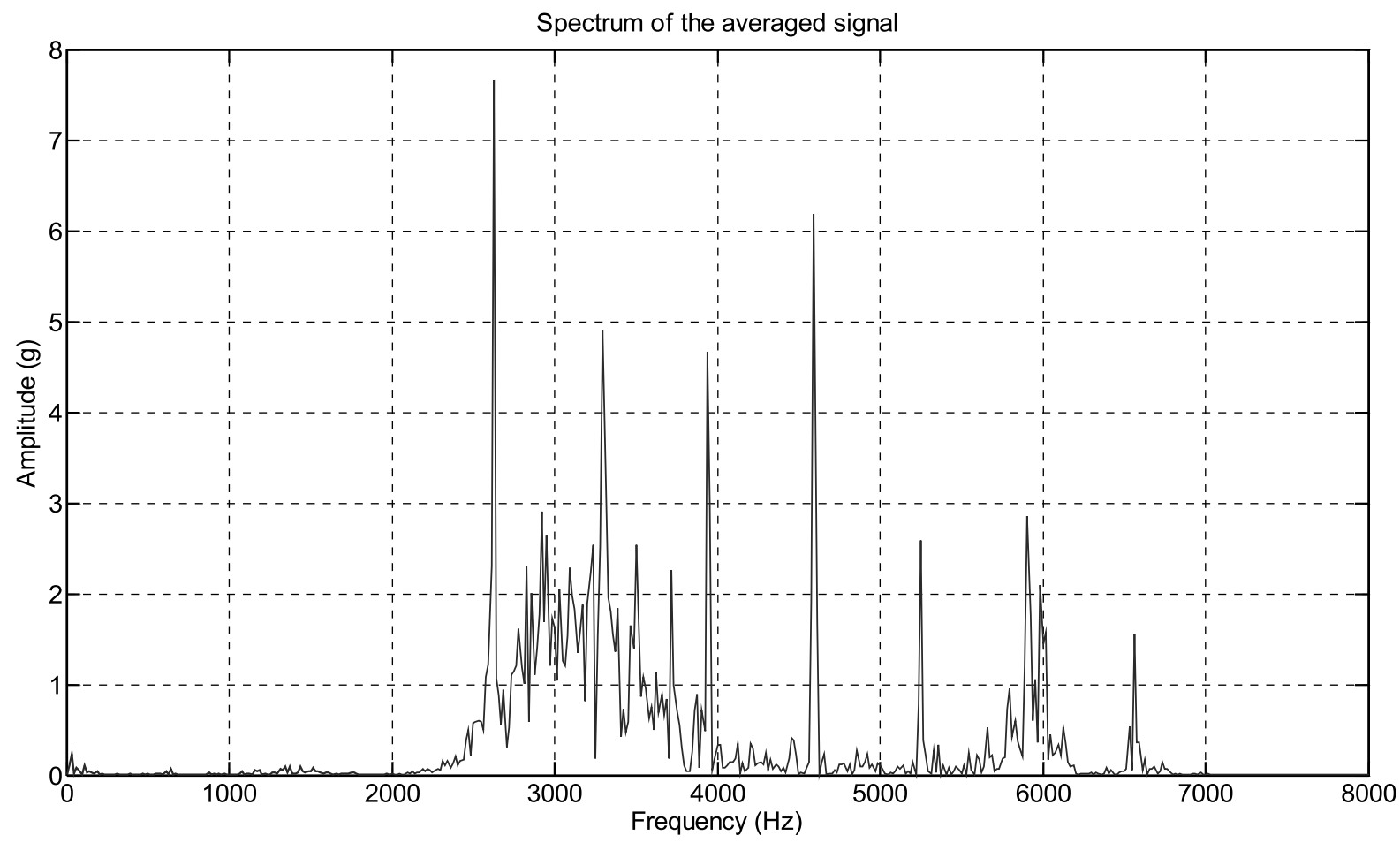

Fig. 22. Frequency spectrum of a fully broken tooth.

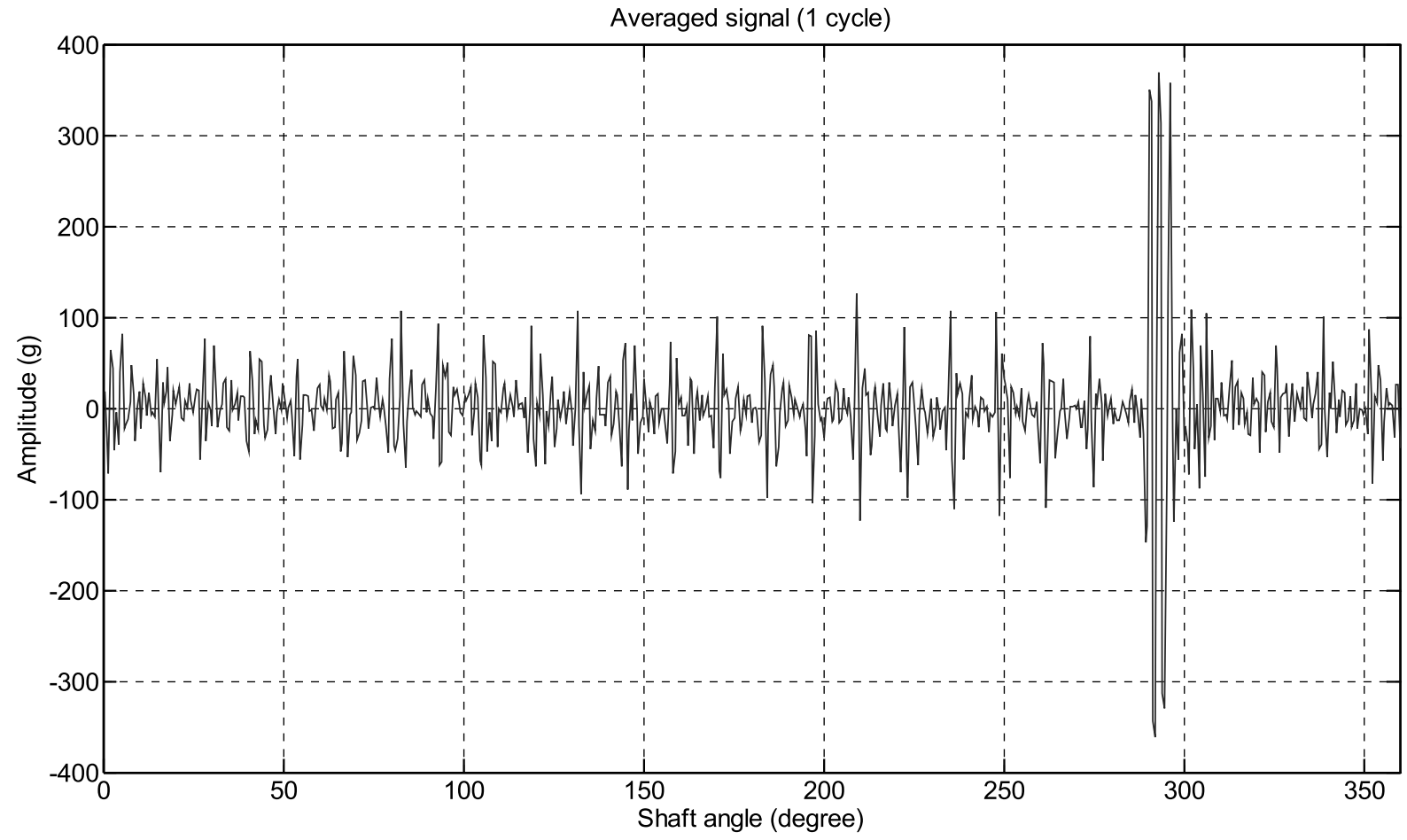

Fig. 23. Time averaged acceleration signal for the fully broken tooth (a complete cycle). 


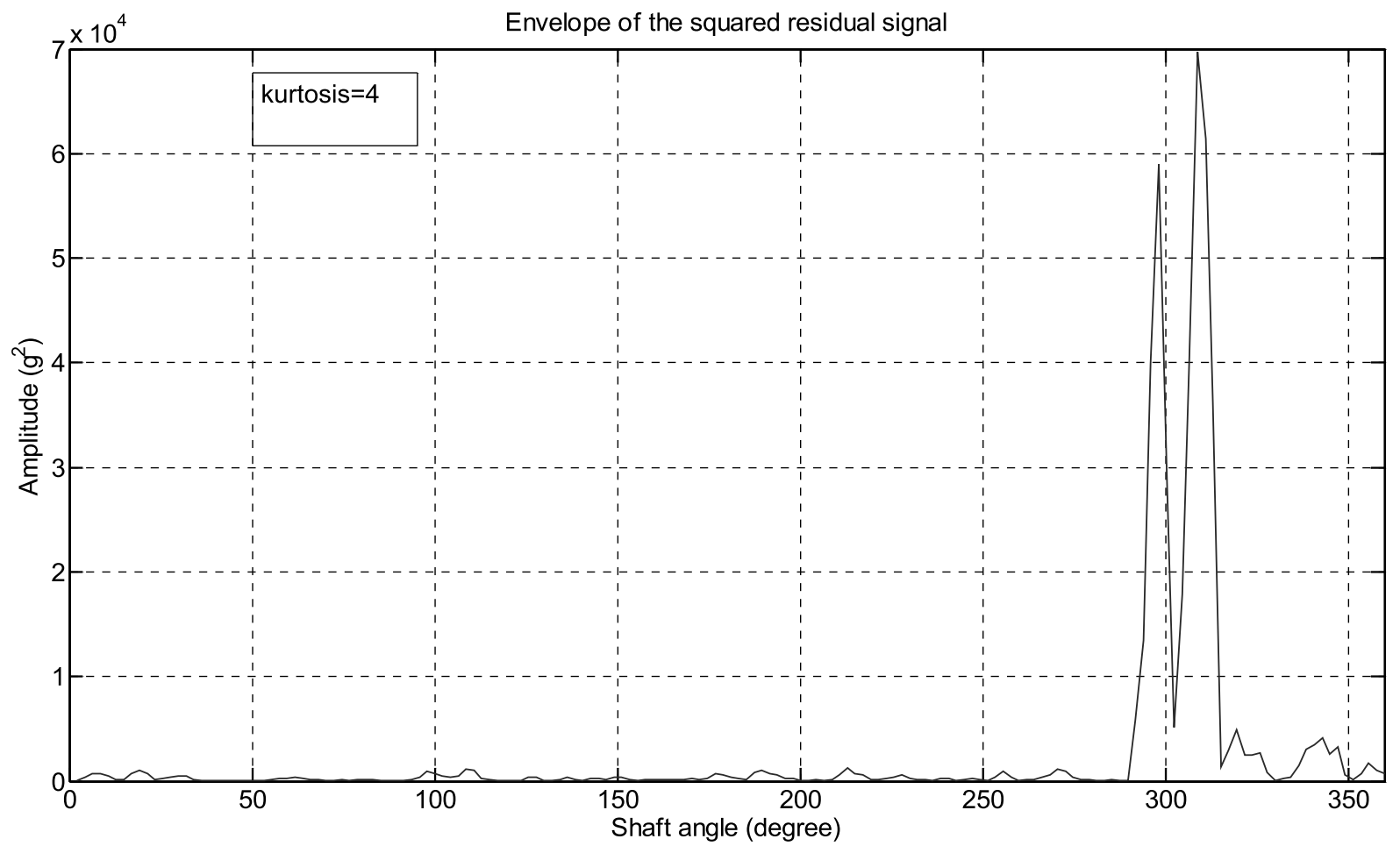

Fig. 24. Envelope of the squared residual signal in the time domain for the fully broken tooth (between 4th and 5th harmonic.

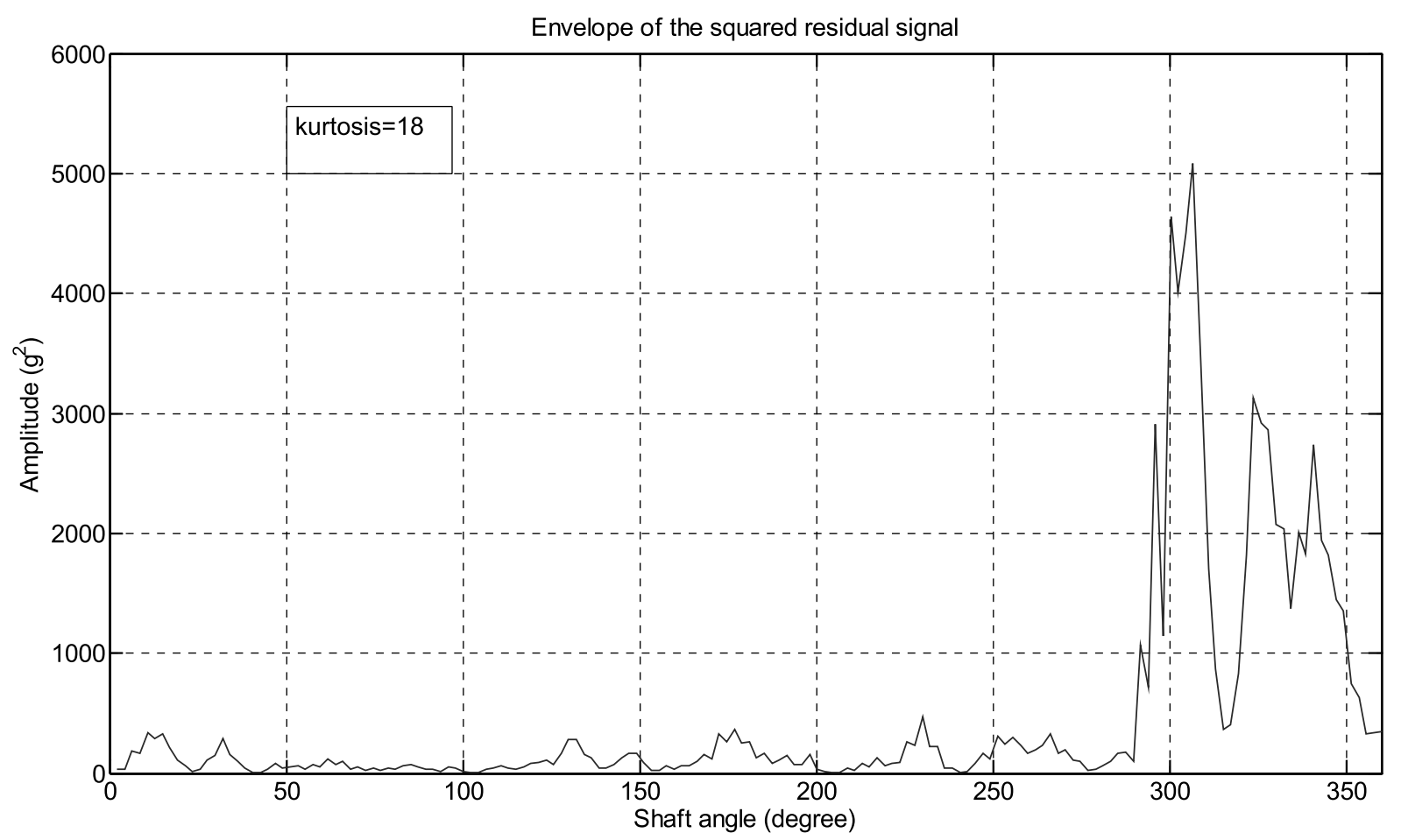

Fig. 25. Envelope of the squared residual signal in the time domain for the fully broken tooth (9th harmonic. 


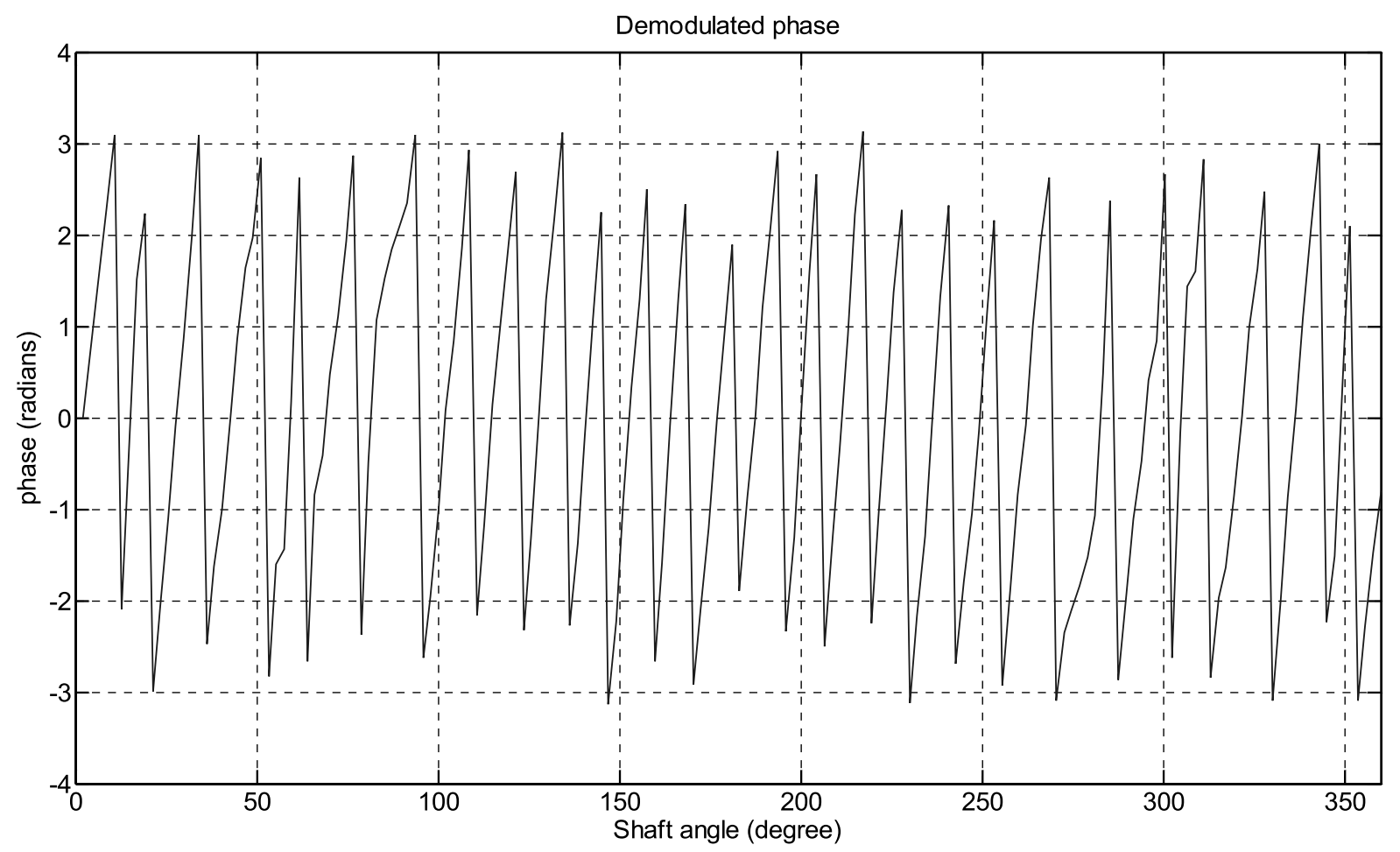

Fig. 26. Phase diagram of the residual signal at the 9th harmonic for the broken tooth.

\section{Acknowledgements}

The authors would like to thank Mr. Sheibaie, Mr. Chitsaz and the modal analysis laboratory at the University of Tabriz for their help and preparing the experimental setup and special thanks goes to Dr. Sadeghi, at Tabriz University for his great help and expert discussions.

\section{References}

[1] N. Baydar and A. Ball, Detection of gear deterioration under varying load conditions by using the instantaneous power spectrum, Journal of Mechanical Systems and Signal Processing 14(6) (2000), 907-921.

[2] N. Baydar and A. Ball, A comparative study of acoustic and vibration signals in detection of gear failures using Wigner-Ville distribution, Journal of Mechanical Systems and Signal Processing 15(6) (2001), 1097-1107.

[3] S. Chitsaz, Gear Box On-Line Diagnosis by Vibration Analysis, MSc. Dissertation, The University of Tabriz, 2005.

[4] L. Cohen, Time Frequency Distribution- A Review, Proceeding of IEEE transaction, 77(7) (1989), 941-981.

[5] G. Dalpiaz, A. Rivola and R. Rubini, Effectiveness and sensitivity of vibration processing techniques for local fault detection in gears, Journal of Mechanical Systems and Signal Processing 14(3) (2000), 387-412.

[6] B.D. Forrester, Use of Wigner-Ville Distribution in Helicopter Transmission Fault Detection, Proceedings of the Australian symposium on signal processing and applications- ASSP, Adelaide, Australia, 1989.

[7] B.D. Forrester, Analysis of Gear Vibration in the Time Frequency Domain: Current Practice and Trends in Mechanical Failure Prevention, Proceeding of the 44th meeting of the mechanical failures prevention group, Willowbrook NJ, 1989, 225-234.

[8] B.E. Halim, M.A.A. Shoukat Choudhury, S.L. Shah and M.J. Zuo, Time domain averaging across all scales: A novel method for detection of gearbox faults, Journal of Mechanical Systems and Signal Processing 22 (2008), 261-278.

[9] R.D. Hippenstiel and P.M. De Olivera, Time-varying spectral estimation using the instantaneous power spectrum, Proceeding of IEEE Transaction 38(10) (1990), 1752-1759.

[10] M. Lebold, K. Mc. Clintic, R. Campbell, C. Byington and K. Maynard, Review of Vibration Analysis Methods for Gearbox diagnostics and Prognostics, Proceeding of the 54th meeting of the society for machinery failure prevention technology, Virginia Beach VA, 2000, 623-634.

[11] M.J. Levin, Instantaneous Spectra and Ambiguity Functions, IEEE transaction on Info theory and IT, 1964, 95-97. 
[12] J. Ma and C.J. Li, On Localized Gear Defect Detection by Demodulation of Vibrations-A Comparative Study, Proceedings of the ASME 1995 International Mechanical Engineering Congress and Exposition, Part 1, San Francisco CA, 1995, 565-576.

[13] J. Ma and C.J. Li, A new Approach to Gear Vibration Demodulation and its Application to Defect Detection, Proceedings for the 48th Meeting of the Mechanical Failures Prevention Group on Advanced Materials and Process Technology for Mechanical Failure Prevention, Wakefield MA, 1994, 43-55.

[14] P.D. Mc. Fadden and W.J. Wang, Early detection of gear failure by vibration analysis- part I: Calculation of the time-frequency distribution, Journal of Mechanical Systems and Signal Processing 7 (1993), 193-203.

[15] P.D. Mc. Fadden, Analysis of the Vibration of the Input bevel Pinion in RAN Wessex Helicopter Main Rotor Gearbox WAK 143prior to Failure, Aero propulsion report 169, DSTO, Australia, 1985.

[16] P.D. Mc. Fadden, Detecting fatigue cracks in gears by amplitude and phase demodulation of the meshing vibration, Journal of Vibration, Acoustics, Stress And Reliability In Design 108(2) (1986), 165-170.

[17] P.D. Mc. Fadden and J.D. Smith, A Signal Processing technique for Detecting Local Defects in Gear from the Signal Average of the Vibration, Proceedings of the institute of mechanical engineers 199, 1985, 287-292.

[18] A.R. Mohanty and C. Kar, Fault Detection in a Multistage Gearbox by Demodulation of Motor Current Waveform, Proceeding of IEEE transaction on Industrial Electronics 53 (2006), 1285-1297.

[19] C. Page, Instantaneous power spectra, Journal of Applied Physics 23(1) (1952), 103-106.

[20] R. Randall, A new method of modeling gear faults, Journal of Mechanical Design 104 (1982), 259-267.

[21] P.D. Samuel and D.J. Pines, A review of vibration-based techniques for helicopter transmission diagnostics, Journal of Sound and Vibration 282 (2005), 475-508.

[22] J.D. Smith, Gear Noise and Vibration (2nd edition), New York, Marcel Dekker Inc., 2003.

[23] C.J. Stander and P.S. Heyns, Using vibration monitoring for local fault detection on gears operating under fluctuating load conditions, Journal of Mechanical Systems and Signal Processing 16(6) (2002), 1005-10024.

[24] W.J. Staszewski, The Application of Time Variant Analysis to Gearbox Fault Detection, Ph.D. Dissertation, University of Manchester, 1994.

[25] W.Q. Wang, I. Fathi and M.F. Golnaraghi, Assessment of gear damage monitoring techniques using vibration measurements, Journal of Mechanical Systems and Signal Processing 15(5) (2001), 905-922.

[26] W.Q. Wang, Early detection of gear tooth cracking using the resonance demodulation technique, Journal of Mechanical Systems and Signal Processing 15(5) (2001), 887-903.

[27] I. Yesilyurt, Fault detection and location in gears by the smoothed instantaneous power spectrum distribution, NDT\&E International Journal 36 (2003), 535-542. 

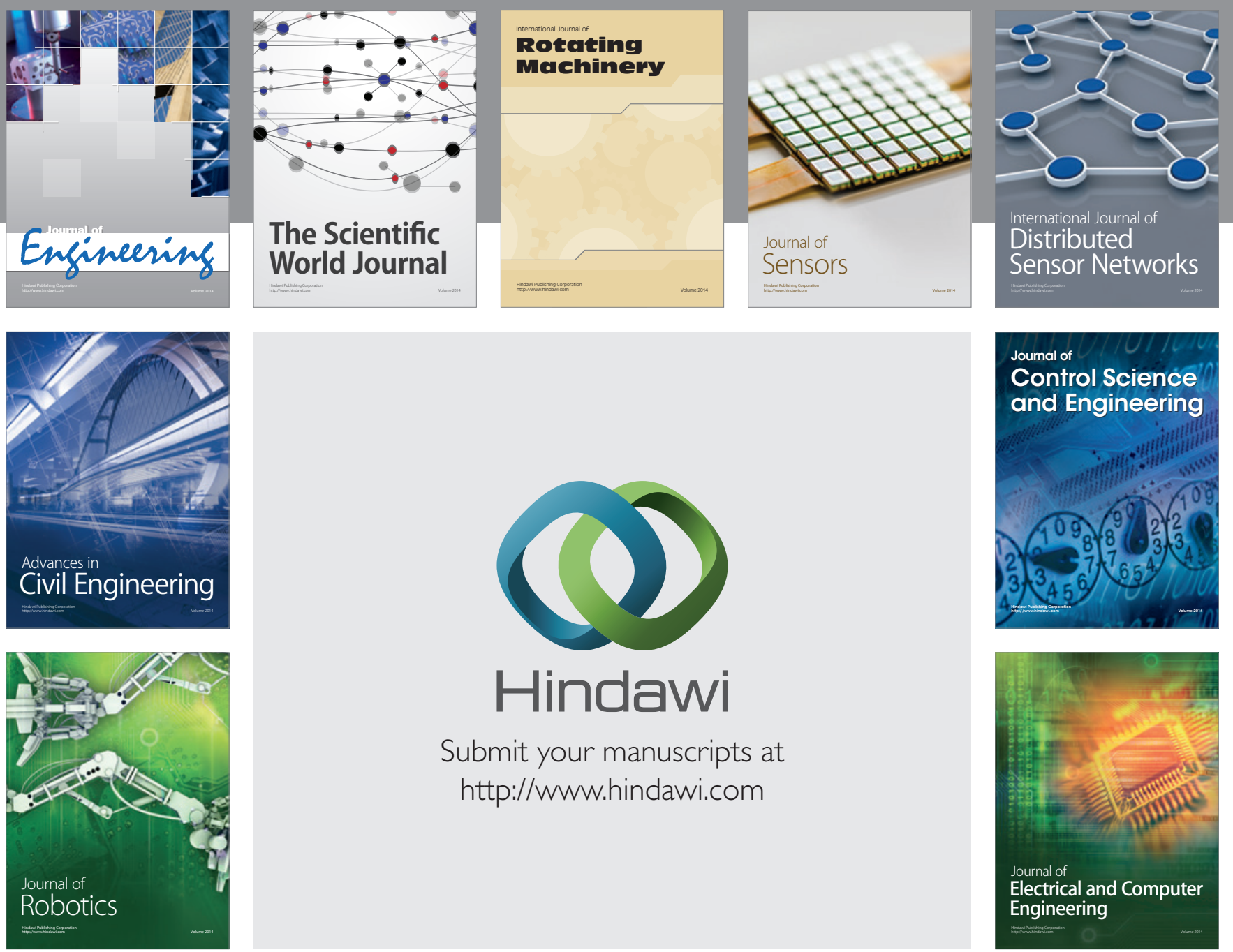

Submit your manuscripts at

http://www.hindawi.com
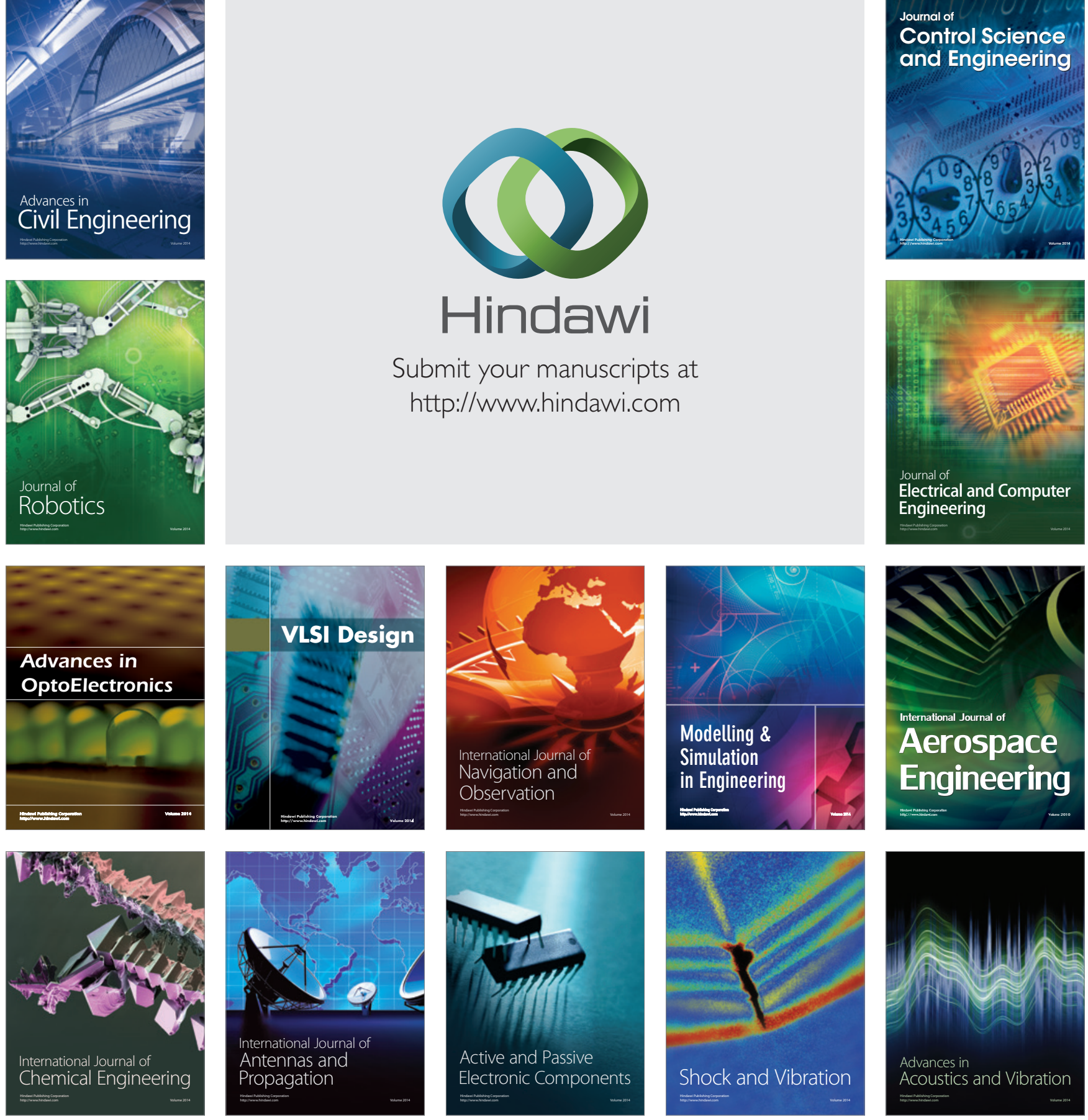